\title{
THE PROPERTIES OF POST-STARBURST QUASARS BASED ON OPTICAL SPECTROSCOPY
}

\author{
Sabrina L. Cales ${ }^{1,2}$, Michael S. Brotherton ${ }^{2}$, Zhaohui Shang ${ }^{2,3}$, Jessie C. Runnoe ${ }^{2}$, Michael A. DiPompeo ${ }^{2}$, \\ Vardha Nicola Bennert ${ }^{4}$, Gabriela Canalizo ${ }^{5,6}$, Kyle D. Hiner $^{5}$, R. Stoll ${ }^{7}$, \\ Rajib Ganguly ${ }^{8}$, and Aleksandar Diamond-Stanic ${ }^{9,10}$ \\ ${ }^{1}$ Departamento de Astronomía, Universidad de Concepción, Casilla 160-C, Concepción, Chile; scales@ astro-udec.cl \\ ${ }^{2}$ Department of Physics and Astronomy, University of Wyoming, Laramie, WY 82071, USA; \\ scales@uwyo.edu,mbrother@uwyo.edu, shang@uwyo.edu, jrunnoe@uwyo.edu,mdipompe@uwyo.edu \\ ${ }^{3}$ Tianjin Normal University, Tianjin 300387, China \\ ${ }^{4}$ Physics Department, California Polytechnic State University, San Luis Obispo, CA 93407, USA; vbennert@ calpoly.edu \\ ${ }^{5}$ Department of Physics and Astronomy, University of California, Riverside, CA 92521, USA; gabriela.canalizo@ucr.edu, khine001@ucr.edu \\ ${ }^{6}$ Institute of Geophysics and Planetary Physics, University of California, Riverside, CA 92521, USA \\ ${ }^{7}$ Department of Astronomy, The Ohio State University, Columbus, OH 43210, USA; stoll@ astronomy.ohio-state.edu \\ ${ }^{8}$ Department of Computer Science, Engineering, \& Physics, University of Michigan-Flint, Flint, MI 48502, USA; ganguly@umflint.edu \\ ${ }^{9}$ Center for Astrophysics and Space Sciences, University of California, San Diego, La Jolla, CA 92093, USA; aleks@ucsd.edu \\ Received 2012 August 12; accepted 2012 November 9; published 2012 December 19
}

\begin{abstract}
We present optical spectroscopy of a sample of 38 post-starburst quasars (PSQs) at $z \sim 0.3,29$ of which have morphological classifications based on Hubble Space Telescope imaging. These broad-lined active galactic nuclei (AGNs) possess the spectral signatures of massive intermediate-aged stellar populations, making them potentially useful for studying connections between nuclear activity and host galaxy evolution. We model the spectra in order to determine the ages and masses of the host stellar populations, and the black hole masses and Eddington fractions of the AGNs. Our model components include an instantaneous starburst, a power law, and emission lines. We find that the PSQs have $M_{\mathrm{BH}} \sim 10^{8} M_{\odot}$ accreting at a few percent of Eddington luminosity and host $\sim 10^{10.5} M_{\odot}$ stellar populations which are several hundred Myr to a few Gyr old. We investigate relationships among these derived properties, spectral properties, and morphologies. We find that PSQs hosted in spiral galaxies have significantly weaker AGN luminosities, older starburst ages, and narrow emission-line ratios diagnostic of ongoing star formation when compared to their early-type counterparts. We conclude that the early-type PSQs are likely the result of major mergers and were likely luminous infrared galaxies in the past, while spiral PSQs with more complex star formation histories are triggered by less dramatic events (e.g., harassment, bars). We provide diagnostics to distinguish the early-type and spiral hosts when high spatial resolution imaging is not available.
\end{abstract}

Key words: galaxies: active - galaxies: evolution - galaxies: interactions - galaxies: Seyfert - galaxies: starburst quasars: general

Online-only material: color figures

\section{INTRODUCTION}

Galaxies harbor supermassive black holes (BHs) at their centers (e.g., Kormendy \& Richstone 1995), the masses of which correlate with that of the host galaxies' bulge component $\left(M_{\mathrm{BH}} \sim 0.15 \% M_{\text {bulge }}\right.$; Merritt \& Ferrarese 2001; Magorrian et al. 1998; Gebhardt et al. 2000a). An even stronger correlation exists between the BH mass and the bulge stellar velocity dispersion (Gebhardt et al. 2000b; Ferrarese \& Merritt 2000; Tremaine et al. 2002). These correlations suggest that BHs and their host galaxies have common evolutionary histories. The nature of the mechanism which is responsible for the "coevolution" of both BH and bulge or even whether only one mechanism is responsible over cosmic time remains unclear. In order to grow to their observed masses, accreting BHs (i.e., active galactic nuclei, AGNs, and/or quasars) are expected to be a phase in the life of every galaxy (Richstone et al. 1998).

A new paradigm involving two mechanisms responsible for mutual BH-bulge growth has been suggested (e.g., Hasinger 2008; Hopkins \& Hernquist 2009; Schawinski et al. 2010b; Bennert et al. 2011). In the early universe, major-merger driven evolution dominates and is responsible for producing the bulk

\footnotetext{
${ }^{10}$ Center for Galaxy Evolution Fellow.
}

of the brightest quasars at $z=2-3$. Below $z \sim 1$, secular evolution and minor interactions become the main fueling mechanisms. Thus, at lower redshifts, less massive systems preferentially show activity (i.e., AGN cosmic downsizing; e.g., Heckman et al. 2004). Furthermore, transitioning from quasar to Seyfert luminosities, fueling rates, and triggering mechanisms may also change (Hopkins \& Hernquist 2009), such that bars in spiral hosts may be sufficient to fuel the nuclear activity of Seyfert galaxies.

In merger-driven evolutionary scenarios, mergers trigger starbursts and the ignition of AGN activity that can in turn inhibit both star formation and its own fueling through feedback (Di Matteo et al. 2005; Springel et al. 2005; Hopkins et al. 2006). A natural consequence of such models is the existence of objects that have luminous quasar activity, starburst, or poststarburst signatures, along with indications of a recent merger, such as tidal debris. Indeed, objects like this do exist, such as UN J1025-0040 which also been called a post-starburst quasar (PSQ; Brotherton et al. 1999; Canalizo et al. 2000; Brotherton et al. 2002).

The post-starburst classification is given for galaxies exhibiting strong Balmer absorption lines, indicating intense star formation in the past $\sim 1 \mathrm{Gyr}$, and a lack of ongoing star formation, as indicated by having little or no nebular emission lines 
(Dressler \& Gunn 1983). Recently, the traditional definition for post-starburst galaxies has been found to be too narrow to encompass the full range in post-starburst populations (Falkenberg et al. 2009). In particular, placing a limit on nebular emission lines (i.e., [O II] 23727) introduces a bias against AGNs (Yan et al. 2006; Wild et al. 2009; Kocevski et al. 2011) and can cause gross underestimation of post-starburst galaxies hosting AGNs since AGNs can power significant emission from [O II].

Several studies of AGN host galaxies in the Sloan Digital Sky Survey (SDSS) have shown that the most luminous AGNs have had a burst of star formation in the past $\sim 1$ Gyr (Vanden Berk et al. 2001; Kauffmann et al. 2003). Additionally, there exists mounting evidence that post-starburst signatures are enhanced in AGNs compared to galaxies which do not exhibit AGN activity (Kocevski et al. 2009; Goto 2006; Georgakakis et al. 2008). In particular, Goto (2006) determines that the fraction of AGNs showing post-starburst features is at least $4.2 \%$, while the fraction of normal galaxies exhibiting these features is $0.2 \%$.

Low-redshift objects hosting the most massive starburst populations and most luminous AGNs may be our best chance at finding the analogs of merger-induced systems at the quasar epoch. Cales et al. (2011, hereafter C11) studied the morphology and disturbance fraction of PSQs via HST/ACS F606W imaging of the most luminous PSQ examples at $z \sim 0.3$ from a spectroscopically selected catalog (Brotherton et al. 2007). C11 find that PSQs are a heterogeneous population of early-type and spiral hosts, with disturbances being equally distributed among the morphologies. The presence of early-type hosts which appear to be major-merger remnants, along with spiral hosts, both isolated and with companions, is suggestive of a more complicated picture than can be explained by galaxy mergers alone. It has become evident that at least two mechanisms are responsible for triggering PSQs, consistent with studies involving mutual $\mathrm{BH}$-bulge growth.

Our follow-up project uses Keck and KPNO $4 \mathrm{~m}$ optical spectroscopy of a sample of 38 PSQs at $z \sim 0.3$. We aim to characterize the $\mathrm{BH}$ masses, Eddington fractions, starburst masses, and starburst ages of PSQs via spectral modeling. Furthermore, 29 of these objects have morphological data from $\mathrm{C} 11$. We continue to characterize the fundamental properties of the AGN and starburst in PSQs and extend the study by investigating the interplay between the properties of PSQs and their morphological subpopulations.

We describe our sample, selection, and data reductions in Section 2. The methodology for decomposing AGNs and starburst stellar populations along with the outputted results and derived fundamental AGNs and post-starburst properties are given in Section 3. We present correlations between fitted and derived properties of the AGN and starburst features in addition to describing how the early-type and spiral host populations differ in Section 4. A summary of our results is given in Section 5. We adopt the cosmology $H_{o}=70 \mathrm{~km} \mathrm{~s}^{-1} \mathrm{Mpc}^{-1}$ and a flat universe where $\Omega_{M}=0.3$ and $\Omega_{\Lambda}=0.7$.

\section{DATA}

\subsection{Sample}

We investigate a sample of 38 objects spectroscopically selected from the Sloan Digital Sky Survey data release 3 (SDSS DR3; Abazajian et al. 2005) that meet the following criteria.

1. Broad emission lines as defined by the SDSS DR3 online database $\left(\right.$ FWHM $\left.>1000 \mathrm{~km} \mathrm{~s}^{-1}\right)$.

2. $r$ model magnitudes $\lesssim 19$.
3. $0.25<z<0.45$.

4. $\mathrm{S} / \mathrm{N}>8$ between rest wavelengths of 4150 and $4250 \AA$ in the SDSS spectra.

5. Summation of the Balmer absorption lines $\mathrm{H} \delta, \mathrm{H} \zeta$, and $\mathrm{H} \eta>2 \AA$ rest-frame equivalent width at a significance greater than $6 \sigma$.

6. $\mathrm{H} \delta>1 \AA$ rest-frame equivalent width.

7. Balmer break $>0.9$; based on the ratio of the fluxes at two $100 \AA$ wide regions centered at rest wavelengths 4035 and $3790 \AA$ A.

The above criteria ensure that all objects in the sample are luminous AGNs with clear post-starburst stellar populations. We call these objects PSQs regardless of whether their AGN component alone exceeds a formal luminosity separating quasar from Seyfert galaxy. Table 1 characterizes some observational properties of the sample. C11 classified morphologies and measured quasar-to-host light contributions based on HST/ACS imaging of a subsample of 29 of these objects. The following sections provide details of our additional ground-based spectroscopy which we obtained in order to improve signal-to-noise ratio $(\mathrm{S} / \mathrm{N})$ and wavelength coverage compared to the SDSS spectra.

\subsection{KPNO Observations}

We obtained long-slit spectra of 12 PSQs and their companions using the Kitt Peak National Observatory (KPNO) $4 \mathrm{~m}$ Mayall telescope on the nights of 2006 May 20-23. We used the $\mathrm{R}-\mathrm{C}$ spectrograph along with the 312 groove $\mathrm{mm}^{-1} \mathrm{KPC}-10 \mathrm{~A}$ grating, in first order, producing a dispersion of $2.75 \AA \mathrm{pixel}^{-1}$ and a resolution of $6.9 \AA \mathrm{FWHM}$ on the TK2B $2048 \times 2048$ CCD detector. The wavelength range covered is 3200 to $7200 \AA$. The detector read noise and gain are $4 e^{-}$and $1.9 e^{-} \mathrm{ADU}^{-1}$, respectively. The long slit is covered at a scale of $0{ }^{\prime} .69$ pixel $^{-1}$; the slit width was $300 \mu \mathrm{m}$ or $2^{\prime \prime}$.

We applied a standard observation strategy with the exception of rotating the slit in order to observe the primary PSQ and its nearest bright companion within $20^{\prime \prime}$ (to be presented in a future publication). We obtained three $1200 \mathrm{~s}$ exposures for each target PSQ. There were three instances where we observed the same target twice, changing the slit angle in order to observe a different companion (i.e., SDSS J115159.59+673604.8, SDSS $\mathrm{J} 123043.41+614821.8$, and SDSS J164444.92+423304.5). In these cases, we obtained six $1200 \mathrm{~s}$ exposures of the primary PSQ. For one source (SDSS J170819.80+603759.4), two of the three $1200 \mathrm{~s}$ exposures had low $\mathrm{S} / \mathrm{N}$ due to poor conditions, and we use the data from a single exposure in our analysis.

Reductions of the spectra were carried out in a standard manner using the IRAF ${ }^{11}$ software. After initial trimming, bias removal, and flat fielding, we extracted the one-dimensional cleaned spectrum using apall. We found the dispersion solution by identifying the lines of an $\mathrm{He}-\mathrm{Ne}-\mathrm{Ar}$ lamp. The spectra were flux calibrated using observations of several spectrophotometric standard stars (Massey et al. 1988). We median combined the individual spectrum with $\mathrm{S} / \mathrm{N}$ weights using scombine. The combinations also include the SDSS spectrum in order to increase the $\mathrm{S} / \mathrm{N}$ and the wavelength coverage of the resulting spectrum. Additional photometric corrections and combinations with other spectra were performed in a similar fashion as the Keck spectra and are described in Section 2.4.

\footnotetext{
11 IRAF is distributed by the National Optical Astronomy Observatory, which is operated by Association of Universities for Research in Astronomy, Inc., under cooperative agreement with the National Science Foundation.
} 
Table 1

Journal of Observations

\begin{tabular}{|c|c|c|c|c|c|c|}
\hline $\begin{array}{l}\text { Object } \\
\text { SDSS }\end{array}$ & $z$ & $r^{\mathrm{a}}$ & $M_{r}^{\mathrm{b}}$ & $\begin{array}{l}\text { Total Int. } \\
\text { Time (s) }\end{array}$ & Spectroscopy & Imaging $^{\mathrm{c}}$ \\
\hline J003043.59-103517.6 & 0.296 & 18.26 & -22.98 & 1200 & Keck & $H S T$ \\
\hline J005739.19+010044.9 & 0.253 & 17.61 & -23.23 & 1200 & Keck & $H S T$ \\
\hline J015259.46+142738.0 & 0.311 & 18.44 & -22.87 & 1200 & Keck & $\ldots$ \\
\hline J020258.94-002807.5 & 0.339 & 18.19 & -23.50 & 1200 & Keck & $H S T$ \\
\hline J021447.00-003250.6 & 0.349 & 18.54 & -23.09 & 1200 & Keck & $H S T$ \\
\hline J023253.42-082832.1 & 0.265 & 17.50 & -23.54 & 1200 & Keck & $\ldots$ \\
\hline J023700.30-010130.5 & 0.344 & 18.58 & -23.05 & 1200 & Keck & $H S T$ \\
\hline J025735.33-001631.3 & 0.362 & 18.67 & -22.95 & 1200 & Keck & $\ldots$ \\
\hline J032143.15-064517.5 & 0.365 & 19.26 & -22.56 & 2400 & Keck & $\ldots$ \\
\hline J040210.90-054630.3 & 0.270 & 18.70 & -22.45 & 1600 & Keck & $H S T$ \\
\hline J074621.06+335040.7 & 0.284 & 17.97 & -23.12 & 1200 & Keck & $H S T$ \\
\hline $\mathrm{J} 075045.00+212546.3$ & 0.408 & 18.01 & -24.18 & 1200 & Keck & $H S T$ \\
\hline $\mathrm{J} 075521.30+295039.2$ & 0.334 & 18.69 & -22.83 & 1200 & Keck & $H S T$ \\
\hline $\mathrm{J} 075549.56+321704.1$ & 0.420 & 18.82 & -23.24 & 2400 & Keck & $H S T$ \\
\hline J081018.67+250921.2 & 0.263 & 17.41 & -23.20 & 300 & Keck & $H S T$ \\
\hline $\mathrm{J} 105816.81+102414.5$ & 0.275 & 18.29 & -22.63 & 3600 & KPNO & $H S T$ \\
\hline J115159.59+673604.8 & 0.274 & 18.44 & -22.64 & 7200 & KPNO & $H S T$ \\
\hline J115355.58+582442.3 & 0.319 & 18.29 & -22.88 & 3600 & KPNO & $H S T$ \\
\hline $\mathrm{J} 123043.41+614821.8$ & 0.324 & 18.63 & -22.77 & 7200 & KPNO & $H S T$ \\
\hline $\mathrm{J} 124833.52+563507.4$ & 0.266 & 17.45 & -23.46 & 3600 & KPNO & $H S T$ \\
\hline $\mathrm{J} 140513.75+625008.2$ & 0.386 & 18.59 & -24.15 & 3600 & KPNO & $\ldots$ \\
\hline $\mathrm{J} 145640.99+524727.2$ & 0.277 & 18.14 & -22.84 & 3600 & KPNO & $H S T$ \\
\hline $\mathrm{J} 145658.15+593202.3$ & 0.326 & 18.58 & -22.86 & 2400 & SDSS & $H S T$ \\
\hline $\mathrm{J} 154534.55+573625.1$ & 0.268 & 18.07 & -22.88 & 3600 & KPNO & $H S T$ \\
\hline J155214.85+565916.9 & 0.335 & 18.49 & -22.97 & 3600 & KPNO & $\ldots$ \\
\hline $\mathrm{J} 164444.92+423304.5$ & 0.317 & 18.98 & -22.16 & 7200 & KPNO & $H S T$ \\
\hline $\mathrm{J} 170046.95+622056.4$ & 0.276 & 18.58 & -22.51 & 3600 & KPNO & $H S T$ \\
\hline J170819.80+603759.4 & 0.289 & 18.92 & -22.19 & 1200 & KPNO & $\ldots$ \\
\hline $\mathrm{J} 210200.42+000501.8$ & 0.329 & 18.16 & -23.38 & 2400 & Keck & $H S T$ \\
\hline $\mathrm{J} 211343.20-075017.6$ & 0.420 & 18.44 & -23.96 & 1200 & Keck & $H S T$ \\
\hline $\mathrm{J} 211838.12+005640.6$ & 0.384 & 18.49 & -23.72 & 1200 & Keck & $H S T$ \\
\hline $\mathrm{J} 212843.42+002435.6$ & 0.346 & 18.78 & -22.92 & 1200 & Keck & $H S T$ \\
\hline J230614.18-010024.4 & 0.267 & 17.77 & -23.22 & 1200 & Keck & $H S T$ \\
\hline $\mathrm{J} 231055.50-090107.6$ & 0.364 & 18.50 & -23.40 & 3600 & Keck & $H S T$ \\
\hline $\mathrm{J} 231317.85-082238.4$ & 0.366 & 18.40 & -23.09 & 1200 & Keck & $\ldots$ \\
\hline $\mathrm{J} 233430.89+140649.7$ & 0.363 & 18.57 & -23.26 & 1200 & Keck & $H S T$ \\
\hline $\mathrm{J} 234335.48-005758.1$ & 0.341 & 18.00 & -23.74 & 1200 & Keck & $\ldots$ \\
\hline $\mathrm{J} 234403.55+154214.0$ & 0.288 & 18.33 & -22.92 & 1200 & Keck & $H S T$ \\
\hline
\end{tabular}

Notes.

a SDSS DR7 $r$ AB magnitudes (modelMag_r).

${ }^{\text {b }}$ SDSS DR7 dereddened $K$-corrected $r$ absolute AB magnitudes.

${ }^{\mathrm{c}}$ Denotes whether morphological data are available from HST ACS/WFC imaging.

\subsection{Keck Observations}

Spectroscopic observations were carried out on 2005 November 1 and 2 with the Low-Resolution Imaging Spectrometer (LRIS; Oke et al. 1995) on the Keck I telescope. For the blue side (LRIS-B), we used the 600 groove $\mathrm{mm}^{-1}$ grism blazed at $4000 \AA$, yielding a dispersion of $0.63 \AA$ pixel $^{-1}$. For the red side (LRIS-R), we used the 400 groove $\mathrm{mm}^{-1}$ grating blazed at $8500 \AA$, yielding a dispersion of $1.86 \AA \mathrm{pixel}^{-1}$. The slit was $1^{\prime \prime}$ wide, projecting to $\sim 7$ pixels on the UV and blue-optimized CCD on LRIS-B and $\sim 5$ pixels on the Tektronix $2048 \times 2048$ CCD on LRIS-R.

We obtained between one or two exposures for each object, typically $1200 \mathrm{~s}$ each, dithering along the slit between exposures. The typical seeing for all observations was 0.7 in $V$. Two or three spectrophotometric standards from Massey et al. (1988) were observed at parallactic angle each night for flux calibration.
The position angles were selected so as to include nearby companion galaxies. The majority of objects were observed at low airmass to minimize the effects of differential atmospheric refraction. The only object that was not observed near transit was SDSS J040210.90-054630.3, which was observed at an airmass of 1.43 .

The spectra were reduced with IRAF, using standard reduction procedures. After subtracting bias, dividing by a normalized halogen lamp flat-field frame and removing sky lines, we rectified the two-dimensional spectra and placed them on a wavelength scale using the least-mean-squares fit of cubic spline segments to identified lines in an $\mathrm{Hg}-\mathrm{Ne}-\mathrm{Cd}-\mathrm{Zn}$ lamp. We calibrated the spectra using the spectrophotometric standards from Massey et al. (1988). The distortions in the spatial coordinate were removed with the IRAF apextract routines. For each slit position, we had two or three individual frames; we averaged the spatially corrected spectra using the IRAF task scombine. 

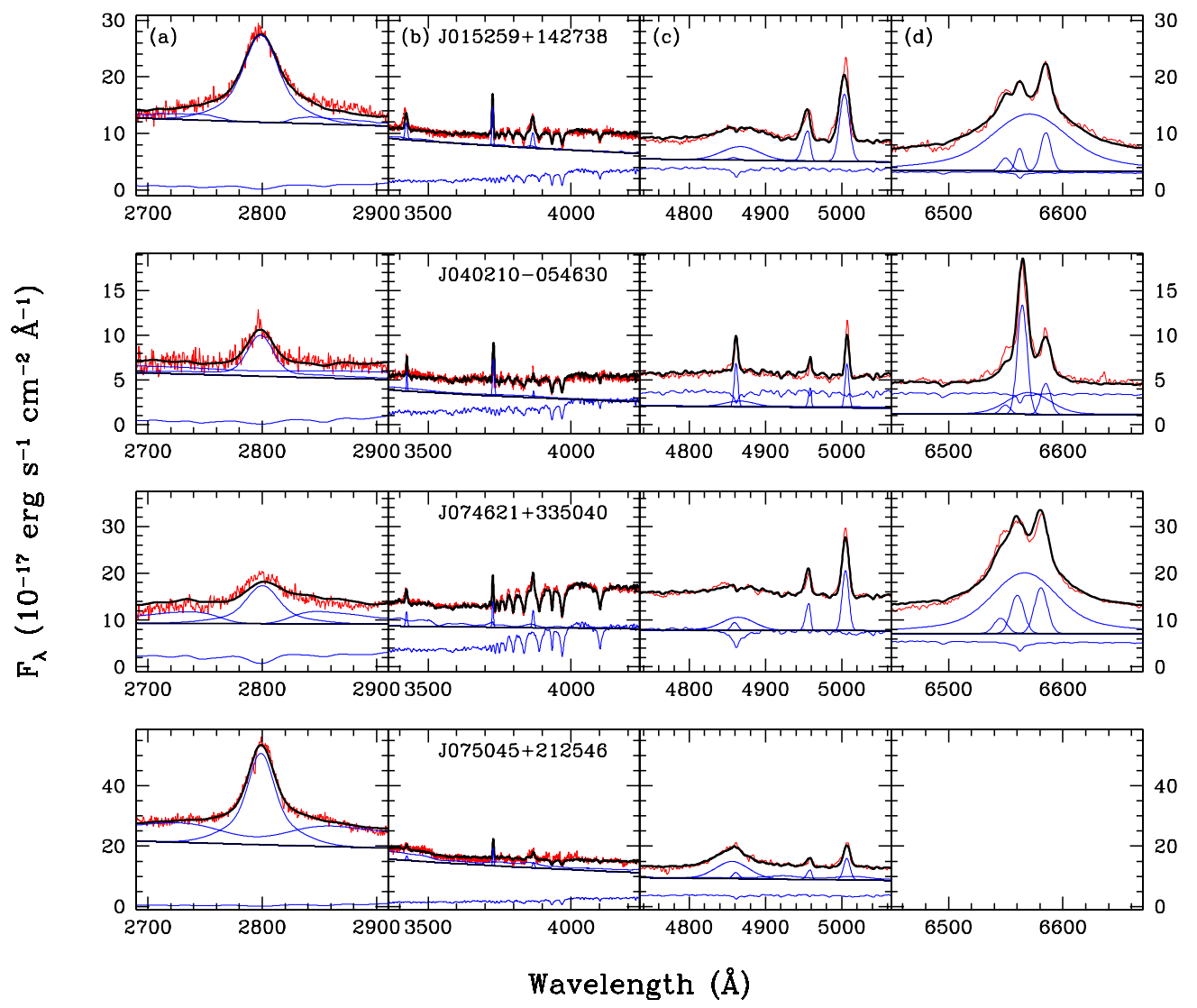

Figure 1. Examples of our spectral decomposition of AGNs and post-starburst stellar population highlighting the (a) $\mathrm{Mg}$ II, (b) Balmer break, (c) $\mathrm{H} \beta$, and (d) $\mathrm{H} \alpha$ regions. The red line is the data. The blue lines make up the components used in the fitting. For ease of interpretation, we plot the emission lines and Fe II templates above the power law. The black line is the model fit to the data.

(A color version of this figure is available in the online journal.)

\subsection{Finalized Spectra and Uncertainties}

Since we chose to orient the slit to also observe neighboring objects we did not observe at parallactic angle. We used the SDSS spectra to correct for atmospheric diffraction slit losses. First, we divide our spectra by the corresponding SDSS spectra and fit the result by a low-order Legendre polynomial. We then corrected our spectra by dividing by the fitted polynomial. The resulting corrected spectra were well matched to the flux calibration of the SDSS spectra.

We corrected for Galactic extinction using the Schlegel et al. (1998) maps and the IRAF task deredden which utilizes the Cardelli et al. (1989) extinction curves. We converted the spectra to rest frame using the IRAF dopcor task using SDSS redshifts. We note that we retained observed fluxes.

We have used a single uniform method to estimate the noise for each spectrum. For each pixel, we compute the root-meansquare value of the difference between the signal and the average using nine pixels centered on the input pixel. We use this estimated noise spectrum to weight individual points in the fitting procedure we describe below.

\section{STELLAR POPULATION SYNTHESIS AND AGN COMPONENT MODELING}

We utilized the IRAF task specfit (Kriss 1994) to model the starburst populations and AGN contributions to the PSQs. The $\chi^{2}$ minimization technique of specfit simultaneously models multiple components. We characterized the AGN contribution of the PSQs using a power law, UV and optical iron emission line blends, as well as multiple Gaussian emission lines from the AGN broad- and narrow-line regions. A Charlot \& Bruzual (S. Charlot \& G. Bruzual 2007, private communication) instantaneous starburst (ISB) varying in age and mass describes the post-starburst component to the PSQs. We characterize the multiple fit components as originating either from the (1) AGN, (2) narrow-line regions, or (3) starburst, and we discuss these components in that order for the remainder of the paper.

We used the same initial guess parameters for each PSQ while changing the step sizes of the power-law index $(0.02,0.05$, and 0.08 ) and starburst age $(50,100$, and $200 \mathrm{Myr})$ corresponding to five runs for each object. We give plots of the runs corresponding to the best fits in the Appendix as well as zoom in on the broad lines and Balmer break for a few of our objects in Figure 1.

Table 2 gives the fitting results for the AGN power law, Fe II templates, and broad lines. The AGN power-law contribution is described by a normalization factor at $1000 \AA$ and the powerlaw index, $\alpha: f_{\lambda} \propto \lambda^{-\alpha}$. The UV and optical iron emission line blends are modeled using the UV and optical Fe II templates derived from I Zw 1 (Vestergaard \& Wilkes 2001; Boroson \& Green 1992). We convolved a Gaussian with the Fe II templates to simulate different velocity widths. The specfit task velocityweight interpolates between the these templates and is also allowed to vary in intensity for each object.

Gaussian emission lines are described by the flux under the profile, line centroid, FWHM, and an asymmetry parameter. We found fitting two broad-line Gaussians to be satisfactory in matching the asymmetries and wings of emission lines 
Table 2

AGN Fitting Results

\begin{tabular}{|c|c|c|c|c|c|c|c|c|c|c|c|c|c|}
\hline \multirow{2}{*}{$\begin{array}{l}\text { Object } \\
\text { SDSSJ }\end{array}$} & \multicolumn{2}{|c|}{ Power Law } & \multicolumn{2}{|c|}{ UV Fe } & \multicolumn{2}{|c|}{$\mathrm{Fe}$ II } & \multicolumn{2}{|c|}{ Mg II } & \multicolumn{2}{|c|}{$\mathrm{H} \beta$} & \multicolumn{2}{|c|}{$\mathrm{H} \alpha$} & \multirow{2}{*}{$\begin{array}{c}\mathrm{H} \alpha \rightarrow \mathrm{H} \beta^{\mathrm{f}} \\
\text { FWHM }\end{array}$} \\
\hline & Norm $^{a}$ & $\alpha^{\mathrm{b}}$ & Scale & FWHM $^{\text {d }}$ & Scale & FWHM & Flux $^{e}$ & FWHM & Flux & FWHM & Flux & FWHM & \\
\hline J003043-103517 & 8.19 & 0.35 & $\ldots g$ & $\ldots$ & $\ldots$ & $\ldots$ & $\ldots$ & $\ldots$ & 106.48 & 7617 & 782.66 & 7926 & 9639 \\
\hline J005739+010044 & 8.09 & 0.28 & $\ldots$ & $\ldots$ & $\ldots$ & $\ldots$ & 175.26 & 7665 & $\ldots$ & $\ldots$ & 497.10 & 4310 & 5084 \\
\hline J015259+142738 & 55.51 & 1.49 & 0.17 & 1792 & $\ldots$ & $\ldots$ & 739.39 & 4128 & 148.29 & 3552 & 1025.44 & 3747 & 4389 \\
\hline J020258-002807 & 4.60 & 0.07 & 0.37 & 9746 & $\ldots$ & $\ldots$ & $\ldots$ & $\ldots$ & $\ldots$ & $\ldots$ & $\ldots$ & $\ldots$ & $\ldots$ \\
\hline J021447-003250 & 17.77 & 0.86 & 0.14 & 3669 & 0.20 & 4770 & 266.95 & 3779 & 88.32 & 5193 & $\ldots$ & $\ldots$ & $\ldots$ \\
\hline J023253-082832 & 16.30 & 0.38 & 0.04 & 11881 & $\ldots$ & $\ldots$ & 478.03 & 5078 & 385.69 & 7077 & 1863.20 & 7596 & 9218 \\
\hline J023700-010130 & 40.81 & 1.96 & 0.48 & 10058 & $\ldots$ & $\ldots$ & 409.88 & 6846 & $\ldots$ & $\ldots$ & $\ldots$ & $\ldots$ & $\ldots$ \\
\hline J025735-001631 & 16.64 & 0.83 & 0.09 & 1855 & 0.36 & 2128 & 268.56 & 2132 & 113.85 & 1872 & $\ldots$ & $\ldots$ & $\ldots$ \\
\hline J032143-064517 & 49.66 & 1.95 & 0.15 & 5515 & $\ldots$ & $\ldots$ & 228.08 & 4469 & 120.45 & 5694 & $\ldots$ & $\ldots$ & $\ldots$ \\
\hline $\mathrm{J} 040210-054630$ & 34.85 & 1.81 & 0.18 & 11729 & $\ldots$ & $\ldots$ & 192.84 & 2943 & 35.12 & 2897 & 131.00 & 2037 & 2315 \\
\hline $\mathrm{J} 074621+335040$ & 16.73 & 0.46 & 0.06 & 5391 & $\ldots$ & $\ldots$ & 374.56 & 3845 & 153.24 & 3079 & 1174.74 & 3244 & 3773 \\
\hline $\mathrm{J} 075045+212546$ & 91.06 & 1.45 & 1.32 & 7961 & 0.69 & 2147 & 1220.57 & 3300 & 310.65 & 3139 & $\ldots$ & $\ldots$ & $\ldots$ \\
\hline J075521+295039 & 6.06 & 0.48 & 0.24 & 9406 & $\ldots$ & $\ldots$ & 188.69 & 2328 & $\ldots$ & $\ldots$ & $\ldots$ & $\ldots$ & $\ldots$ \\
\hline J075549+321704 & 43.29 & 1.49 & 0.38 & 8578 & 0.21 & 4715 & 267.67 & 3794 & 172.21 & 4505 & $\ldots$ & $\ldots$ & $\ldots$ \\
\hline J081018+250921 & 114.36 & 1.06 & 4.24 & 9618 & 2.05 & 9477 & 6808.27 & 8397 & 1100.02 & 5259 & 1682.93 & 4357 & 5142 \\
\hline $\mathrm{J} 105816+102414$ & 47.41 & 1.40 & $\ldots$ & $\ldots$ & $\ldots$ & $\ldots$ & 660.14 & 8426 & 310.05 & 9099 & 857.88 & 4683 & 5548 \\
\hline J115159+673604 & 7.25 & 0.82 & $\ldots$ & $\ldots$ & $\ldots$ & $\ldots$ & $\ldots$ & $\ldots$ & $\ldots$ & $\ldots$ & 845.27 & 4781 & 5669 \\
\hline $\mathrm{J} 115355+582442$ & 24.73 & 1.19 & 0.54 & 3850 & 0.52 & 4799 & 544.21 & 2837 & 125.73 & 2710 & 541.56 & 3466 & 4044 \\
\hline $\mathrm{J} 123043+614821$ & 28.25 & 1.16 & 0.64 & 8865 & 0.28 & 4687 & 753.57 & 3464 & 84.21 & 3623 & 990.86 & 4226 & 4980 \\
\hline J124833+563507 & 7.90 & 0.22 & 0.39 & 5999 & 0.76 & 4237 & $\ldots$ & $\ldots$ & 193.10 & 4645 & 1351.19 & 5125 & 6098 \\
\hline $\mathrm{J} 140513+625008$ & 3.84 & 0.23 & 0.23 & 8434 & 0.22 & 5496 & 543.69 & 4336 & $\ldots$ & $\ldots$ & 558.75 & 4505 & 5325 \\
\hline $\mathrm{J} 145640+524727$ & 11.55 & 0.75 & 0.36 & 10286 & 0.22 & 4331 & $\ldots$ & $\ldots$ & 79.52 & 4084 & 353.92 & 3266 & 3800 \\
\hline J145658+593202 & 5.03 & 0.53 & $\ldots$ & $\ldots$ & $\ldots$ & $\ldots$ & $\ldots$ & $\ldots$ & 55.34 & 4686 & 453.90 & 6826 & 8240 \\
\hline $\mathrm{J} 154534+573625$ & 45.24 & 1.13 & 0.47 & 11684 & $\ldots$ & $\ldots$ & 1257.66 & 8640 & 288.11 & 10263 & 2144.13 & 8093 & 9852 \\
\hline J155214+565916 & 10.71 & 0.71 & 0.20 & 10251 & $\ldots$ & $\ldots$ & $\ldots$ & $\ldots$ & 61.05 & 3830 & 334.88 & 5032 & 5982 \\
\hline $\mathrm{J} 164444+423304$ & 11.05 & 0.75 & 0.56 & 11129 & 0.27 & 6715 & 200.53 & 2503 & 58.49 & 2512 & 319.85 & 2482 & 2848 \\
\hline $\mathrm{J} 170046+622056$ & 38.50 & 1.67 & $\ldots$ & $\ldots$ & $\ldots$ & $\ldots$ & 1098.66 & 9929 & $\ldots$ & $\ldots$ & 900.31 & 6724 & 8109 \\
\hline $\mathrm{J} 170819+603759$ & 8.56 & 0.64 & $\ldots$ & $\ldots$ & $\ldots$ & $\ldots$ & $\ldots$ & $\ldots$ & 89.78 & 5173 & $\ldots$ & $\ldots$ & $\ldots$ \\
\hline $\mathrm{J} 210200+000501$ & 136.70 & 2.35 & 0.45 & 11819 & $\ldots$ & $\ldots$ & 883.42 & 4471 & 138.74 & 4330 & $\ldots$ & $\ldots$ & $\ldots$ \\
\hline $\mathrm{J} 211343-075017$ & 101.42 & 2.16 & $\ldots$ & $\ldots$ & $\ldots$ & $\ldots$ & 1525.69 & 5977 & 185.33 & 7055 & $\ldots$ & $\ldots$ & $\ldots$ \\
\hline $\mathrm{J} 211838+005640$ & 46.35 & 1.58 & 0.32 & 4175 & $\ldots$ & $\ldots$ & 645.84 & 4539 & 201.13 & 7200 & $\ldots$ & $\ldots$ & $\ldots$ \\
\hline $\mathrm{J} 212843+002435$ & 13.22 & 0.49 & 0.00 & 3671 & $\ldots$ & $\ldots$ & 363.04 & 2983 & 165.69 & 3203 & $\ldots$ & $\ldots$ & $\ldots$ \\
\hline J230614-010024 & 10.43 & 0.25 & $\ldots$ & $\ldots$ & $\ldots$ & $\ldots$ & 412.81 & 4728 & 156.91 & 4440 & 651.67 & 2688 & 3097 \\
\hline J231055-090107 & 50.07 & 2.10 & 0.43 & 9152 & $\ldots$ & $\ldots$ & 805.11 & 5628 & $\ldots$ & $\ldots$ & $\ldots$ & $\ldots$ & $\ldots$ \\
\hline $\mathrm{J} 231317-082238$ & 11.75 & 0.57 & 0.25 & 3190 & 0.25 & 4478 & 430.44 & 4872 & 137.26 & 4322 & $\ldots$ & $\ldots$ & $\ldots$ \\
\hline $\mathrm{J} 233430+140649$ & 15.78 & 0.51 & 0.34 & 10045 & $\ldots$ & $\ldots$ & 588.16 & 6233 & 174.42 & 6635 & $\ldots$ & $\ldots$ & $\ldots$ \\
\hline $\mathrm{J} 234335-005758$ & 3.46 & 0.07 & $\ldots$ & $\ldots$ & $\ldots$ & $\ldots$ & $\ldots$ & $\ldots$ & 45.45 & 7095 & $\ldots$ & $\ldots$ & $\ldots$ \\
\hline $\mathrm{J} 234403+154214$ & 31.76 & 1.98 & 0.55 & 9162 & $\ldots$ & $\ldots$ & 423.99 & 4664 & 6.14 & 20023 & 746.51 & 4393 & 5187 \\
\hline
\end{tabular}

Notes.

a The flux at $1000 \AA$, in units of $10^{-17} \mathrm{erg} \mathrm{s}^{-1} \mathrm{~cm}^{-2} \AA^{-1}$.

b Power-law index.

c The factor by which the Fe II templates have been scaled.

${ }^{\mathrm{d}} \mathrm{FWHM}$ is given in rest frame $\mathrm{km} \mathrm{s}^{-1}$.

e Scaling for the Gaussian emission lines corresponds to the integrated area under the curve as flux in units of $10^{-17} \mathrm{erg} \mathrm{s}^{-1} \mathrm{~cm}^{-2}$.

${ }^{\mathrm{f}}$ Estimated values of $\mathrm{H} \beta$ based on $\mathrm{H} \alpha$ measurements using the relation given by Shen et al. (2011).

g Denotes discarded measurements or no coverage.

originating from the broad-line region (i.e., $\mathrm{Mg}$ II, $\mathrm{H} \beta$, and $\mathrm{H} \alpha$ ). Thus, we held the asymmetry parameter at "symmetric" and include two broad Gaussians in order to better model line asymmetries. We obtain a single broad-line FWHM by numerically finding the FWHM of the superposition of the two Gaussians. In order to separate the narrow and broad lines and also to characterize other narrow lines of interest, we included a single Gaussian for each of the narrow lines; [Ne v] $\lambda 3426$, [O II] $\lambda 3727$, [Ne III] $\lambda 3869, \mathrm{H} \beta \lambda 4861,[\mathrm{O}$ III] $\lambda 4959$, [O III] $\lambda 5007$, [ $\mathrm{N}_{\text {II }} \lambda \lambda_{6548, \mathrm{H} \alpha} \lambda 6563$, and [N II] $\lambda 6583$. Tables 3 and 4 give the fitting results for the narrow lines.

The ISB modeling technique assumes that all stars are coeval with the same chemical composition. We used the solar metallicity models of S. Charlot \& G. Bruzual (in preparation) and a Chabrier (2003) initial mass function (IMF) to model the starburst contribution and find the best fit by varying the starburst age and intensity. The specfit task age-weight interpolates between the stellar population ages of 56, 75, 100, 133, 177, 237, 316, 422, 562, 750, 1000, 1330, 1770, and 2370 Myr. Our age estimates are insensitive to changes in the IMF. We discuss starburst mass calculations in Section 3.3. Table 5 gives fitting results for the post-starburst stellar populations.

After choosing the fit corresponding to the best $\chi^{2}$, we analyzed the goodness of fit by eye for each object and interactively made adjustments to the fit parameters as necessary. We discarded components where the $\mathrm{S} / \mathrm{N}$ was low $(\leqslant 5$; particularly troublesome for $\mathrm{Mg}$ II and in some objects the UV Fe II templates) and in the complicated $\mathrm{H} \beta$ region where degeneracies 
Table 3

Narrow-line Fitting Results-A

\begin{tabular}{|c|c|c|c|c|c|c|c|c|c|c|c|c|}
\hline \multirow{2}{*}{$\begin{array}{l}\text { Object } \\
\text { SDSSJ }\end{array}$} & \multicolumn{2}{|c|}{$[\mathrm{Ne} \mathrm{V}]$} & \multicolumn{2}{|c|}{$[\mathrm{O} \mathrm{II}]$} & \multicolumn{2}{|c|}{ [Ne III] } & \multicolumn{2}{|c|}{$\mathrm{H} \beta$} & \multicolumn{2}{|c|}{ [O III] $\lambda 4959$} & \multicolumn{2}{|c|}{$[\mathrm{O} \mathrm{III}] \lambda 5007$} \\
\hline & Flux $^{a}$ & FWHM $^{\mathrm{b}}$ & Flux & FWHM & Flux & FWHM & Flux & FWHM & Flux & FWHM & Flux & FWHM \\
\hline J003043-103517 & 9.29 & 798 & 51.62 & 731 & 21.77 & 798 & 30.58 & 798 & 22.19 & 798 & 66.56 & 798 \\
\hline J005739+010044 & 6.95 & 469 & 24.64 & 411 & 11.47 & 469 & 43.85 & 469 & 12.46 & 469 & 37.37 & 469 \\
\hline J015259+142738 & 33.24 & 898 & 41.37 & 449 & 32.15 & 898 & 6.78 & 898 & 63.05 & 898 & 189.16 & 898 \\
\hline J020258-002807 & 7.93 & 590 & 152.68 & 562 & -1.13 & 590 & 78.70 & 590 & 20.24 & 590 & 60.71 & 590 \\
\hline J021447-003250 & 13.65 & 752 & 28.59 & 440 & 15.42 & 752 & 27.79 & 752 & 28.98 & 752 & 86.95 & 752 \\
\hline J023253-082832 & 15.32 & 699 & 36.25 & 754 & 21.02 & 699 & 11.25 & 699 & 18.73 & 699 & 56.18 & 699 \\
\hline J023700-010130 & 13.42 & 503 & 81.27 & 523 & 3.20 & 503 & 53.77 & 503 & 31.65 & 503 & 94.94 & 503 \\
\hline J025735-001631 & 19.29 & 517 & 28.07 & 458 & 11.16 & 517 & 16.15 & 517 & 32.66 & 517 & 97.98 & 517 \\
\hline J032143-064517 & 9.26 & 621 & 7.73 & 792 & 16.88 & 621 & $\ldots$ & $\ldots$ & 14.29 & 621 & 42.86 & 621 \\
\hline J040210-054630 & 3.55 & 348 & 32.00 & 586 & 9.23 & 348 & 29.15 & 348 & 10.16 & 348 & 30.49 & 348 \\
\hline $\mathrm{J} 074621+335040$ & 31.47 & 612 & 32.46 & 449 & 22.90 & 612 & 17.67 & 612 & 46.58 & 612 & 139.75 & 612 \\
\hline J075045+212546 & 15.45 & 608 & 30.39 & 389 & 11.46 & 608 & 21.34 & 608 & 25.65 & 608 & 76.96 & 608 \\
\hline J075521+295039 & 7.69 & 398 & 55.86 & 426 & 4.64 & 398 & 41.49 & 398 & 22.17 & 398 & 66.52 & 398 \\
\hline J075549+321704 & 16.08 & 695 & 19.61 & 736 & 10.84 & 695 & 18.09 & 695 & 24.20 & 695 & 72.60 & 695 \\
\hline J081018+250921 & 23.92 & 532 & $\ldots$ & $\ldots$ & 36.57 & 532 & $\ldots$ & $\ldots$ & 65.71 & 532 & 197.13 & 532 \\
\hline $\mathrm{J} 105816+102414$ & 13.79 & 429 & 38.29 & 943 & 15.22 & 429 & 17.97 & 429 & 42.88 & 429 & 128.63 & 429 \\
\hline J115159+673604 & 9.49 & 584 & 23.87 & 507 & 6.38 & 584 & 13.60 & 584 & 15.66 & 584 & 46.97 & 584 \\
\hline $\mathrm{J} 115355+582442$ & 18.44 & 659 & 58.94 & 572 & 5.89 & 659 & 19.63 & 659 & 36.92 & 659 & 110.75 & 659 \\
\hline $\mathrm{J} 123043+614821$ & 8.49 & 449 & 41.79 & 553 & 10.41 & 449 & 23.88 & 449 & 12.83 & 449 & 38.48 & 449 \\
\hline $\mathrm{J} 124833+563507$ & $\ldots$ & $\ldots$ & 17.68 & 440 & $\ldots$ & $\ldots$ & $\ldots$ & $\ldots$ & $\ldots$ & $\ldots$ & $\ldots$ & $\ldots$ \\
\hline $\mathrm{J} 140513+625008$ & 22.15 & 798 & 30.04 & 617 & 14.63 & 798 & 11.82 & 798 & 24.66 & 798 & 73.97 & 798 \\
\hline $\mathrm{J} 145640+524727$ & $\ldots$ & $\ldots$ & 23.00 & 490 & $\ldots$ & $\ldots$ & 6.38 & 200 & $\ldots$ & $\ldots$ & $\ldots$ & $\ldots$ \\
\hline J145658+593202 & 11.02 & 384 & 50.49 & 516 & 7.15 & 384 & 22.66 & 384 & 21.31 & 384 & 63.92 & 384 \\
\hline $\mathrm{J} 154534+573625$ & 43.09 & 561 & 157.47 & 728 & 28.11 & 561 & 47.83 & 561 & 100.47 & 561 & 301.40 & 561 \\
\hline J155214+565916 & 4.42 & 328 & 34.27 & 606 & 3.84 & 328 & 18.71 & 328 & 10.31 & 328 & 30.94 & 328 \\
\hline J164444+423304 & $\ldots$ & $\ldots$ & 28.66 & 779 & 1.55 & 691 & 16.94 & 691 & 10.82 & 691 & 32.45 & 691 \\
\hline $\mathrm{J} 170046+622056$ & 23.89 & 753 & 41.60 & 1144 & 16.56 & 753 & 34.35 & 753 & 18.11 & 753 & 54.33 & 753 \\
\hline J170819+603759 & $\ldots$ & $\ldots$ & $\ldots$ & $\ldots$ & $\ldots$ & $\ldots$ & 28.04 & 716 & 26.50 & 716 & 79.51 & 716 \\
\hline $\mathrm{J} 210200+000501$ & 23.88 & 814 & 50.77 & 631 & 2.93 & 814 & 4.95 & 814 & 11.97 & 814 & 35.91 & 814 \\
\hline $\mathrm{J} 211343-075017$ & 38.08 & 688 & 55.43 & 538 & 25.91 & 688 & $\ldots$ & $\ldots$ & 47.50 & 688 & 142.51 & 688 \\
\hline $\mathrm{J} 211838+005640$ & 19.99 & 671 & 22.14 & 450 & 11.42 & 671 & 9.02 & 671 & 46.84 & 671 & 140.51 & 671 \\
\hline $\mathrm{J} 212843+002435$ & 11.07 & 642 & 14.59 & 693 & 10.13 & 642 & 5.04 & 642 & 16.70 & 642 & 50.10 & 642 \\
\hline $\mathrm{J} 230614-010024$ & 11.91 & 344 & 61.32 & 473 & 6.99 & 344 & 69.20 & 344 & 21.36 & 344 & 64.08 & 344 \\
\hline J231055-090107 & 17.28 & 674 & 56.33 & 627 & 10.46 & 674 & 25.68 & 674 & 20.59 & 674 & 61.77 & 674 \\
\hline $\mathrm{J} 231317-082238$ & 9.32 & 794 & 9.00 & 300 & 5.34 & 794 & 8.13 & 794 & 17.92 & 794 & 53.76 & 794 \\
\hline $\mathrm{J} 233430+140649$ & 30.54 & 689 & 140.41 & 525 & 15.50 & 689 & 55.65 & 689 & 64.98 & 689 & 194.94 & 689 \\
\hline $\mathrm{J} 234335-005758$ & 8.37 & 603 & 42.66 & 818 & 6.16 & 603 & 35.25 & 603 & 16.57 & 603 & 49.71 & 603 \\
\hline $\mathrm{J} 234403+154214$ & $\ldots$ & $\ldots$ & 10.98 & 729 & $\ldots$ & $\ldots$ & $\ldots$ & $\ldots$ & $\ldots$ & $\ldots$ & $\ldots$ & $\ldots$ \\
\hline
\end{tabular}

Notes. The FWHM of the narrow emission lines should not be considered physical due to the low spectral resolution of our KPNO and Keck spectra.

a Scaling for the Gaussian emission lines corresponds to the integrated area under the curve as flux in units of $10^{-17} \mathrm{erg} \mathrm{s}^{-1} \mathrm{~cm}^{-2}$.

${ }^{\mathrm{b}} \mathrm{FWHM}$ is given in rest frame $\mathrm{km} \mathrm{s}^{-1}$.

may exist between the emission of $\mathrm{H} \beta+[\mathrm{O}$ III $] \lambda 4959+[\mathrm{O}$ III $]$ $\lambda 5007$ and the stellar absorption at $\mathrm{H} \beta$. We also note that for some objects the iron emission from I Zw 1 does not match the observed iron emission on the blue side of $\mathrm{Mg}$ II. This mismatch is understood to be a result of different relative contributions to the multiplets giving rise to the iron emission. The fits to these objects would be greatly improved if we simply doubled the flux of the template redward of $3025 \AA$.

We note that the HST photometry of C11 matches the AGN power-law continuum to within $25 \%$ in flux. This is in general good agreement considering variability issues and aperture differences.

\subsection{Caveats about Spectral Modeling}

While our spectral fits are good in general, our model assumptions are too simple in some respects and their effects on our results should be considered more closely. In particular, metallicity can vary between galaxies and within galaxies. Dust, often associated with starbursts and sometimes AGNs, can also be present along the line of sight and affect model parameters. Finally, the original starburst event we model as an instantaneous burst certainly takes place in the environment of one or more galaxies with existing, older stellar populations. Ongoing star formation may be present as well. We briefly discuss these points below. However, each of these may represent more complex problems requiring more and higher $\mathrm{S} / \mathrm{N}$ data to be more fully explored in future investigations.

Metallicity affects the spectra of stars. If we assumed a metallicity higher than solar we would fit a stellar population that is younger and less massive. Conversely, assuming a lower metallicity would result in our fitting a stellar population that is older and more massive. In the case of the PSQ prototype UN J1025-0040, the uncertainty in the age of the $400 \mathrm{Myr}$ old stellar population was $\pm 50 \mathrm{Myr}$ assuming a reasonable range in metallicity (Brotherton et al. 1999). Their methodology is similar to ours and we expect similar uncertainties. 
Table 4

Narrow-line Fitting Results-B

\begin{tabular}{|c|c|c|c|c|c|c|}
\hline \multirow{2}{*}{$\begin{array}{l}\text { Object } \\
\text { SDSSJ }\end{array}$} & \multicolumn{2}{|c|}{$\left[\mathrm{N}_{\mathrm{II}}\right] \lambda 6548$} & \multicolumn{2}{|c|}{$\mathrm{H} \alpha$} & \multicolumn{2}{|c|}{$\left[\mathrm{N}_{\mathrm{II}}\right] \lambda 6583$} \\
\hline & Flux $^{a}$ & FWHM $^{\mathrm{b}}$ & Flux & FWHM & Flux & FWHM \\
\hline J003043-103517 & 37.67 & 694 & 122.86 & 694 & 113.01 & 694 \\
\hline J005739+010044 & 33.70 & 469 & 224.43 & 469 & 101.10 & 469 \\
\hline J015259+142738 & 26.10 & 493 & 32.62 & 354 & 78.29 & 493 \\
\hline J020258-002807 & $\ldots$ & $\ldots$ & $\ldots$ & $\ldots$ & $\ldots$ & $\ldots$ \\
\hline J021447-003250 & $\ldots$ & $\ldots$ & $\ldots$ & $\ldots$ & $\ldots$ & $\ldots$ \\
\hline J023253-082832 & 21.08 & 699 & 102.62 & 699 & 63.24 & 699 \\
\hline J023700-010130 & $\ldots$ & $\ldots$ & $\ldots$ & $\ldots$ & $\ldots$ & $\ldots$ \\
\hline J025735-001631 & $\ldots$ & $\ldots$ & $\ldots$ & $\ldots$ & $\ldots$ & $\ldots$ \\
\hline J032143-064517 & $\ldots$ & $\ldots$ & $\ldots$ & $\ldots$ & $\ldots$ & $\ldots$ \\
\hline J040210-054630 & 11.00 & 456 & 130.00 & 456 & 37.00 & 456 \\
\hline $\mathrm{J} 074621+335040$ & 46.50 & 612 & 117.05 & 612 & 139.49 & 612 \\
\hline $\mathrm{J} 075045+212546$ & $\ldots$ & $\ldots$ & $\ldots$ & $\ldots$ & $\ldots$ & $\ldots$ \\
\hline J075521+295039 & $\ldots$ & $\ldots$ & $\ldots$ & $\ldots$ & $\ldots$ & $\ldots$ \\
\hline J075549+321704 & $\ldots$ & $\ldots$ & $\ldots$ & $\ldots$ & $\ldots$ & $\ldots$ \\
\hline J081018+250921 & 17.00 & 532 & 11.33 & 532 & 51.01 & 532 \\
\hline $\mathrm{J} 105816+102414$ & 20.33 & 429 & 49.17 & 429 & 60.98 & 429 \\
\hline $\mathrm{J} 115159+673604$ & 12.48 & 295 & 41.61 & 295 & 37.44 & 295 \\
\hline J115355+582442 & 35.49 & 659 & 88.45 & 659 & 106.48 & 659 \\
\hline $\mathrm{J} 123043+614821$ & 41.68 & 449 & 217.61 & 449 & 125.04 & 449 \\
\hline $\mathrm{J} 124833+563507$ & 25.94 & 513 & 83.91 & 513 & 77.81 & 513 \\
\hline $\mathrm{J} 140513+625008$ & 18.78 & 798 & 37.76 & 798 & 56.34 & 798 \\
\hline $\mathrm{J} 145640+524727$ & 9.42 & 200 & 44.01 & 200 & 28.26 & 200 \\
\hline $\mathrm{J} 145658+593202$ & 17.87 & 384 & 97.65 & 384 & 53.62 & 384 \\
\hline $\mathrm{J} 154534+573625$ & 92.60 & 561 & 211.54 & 561 & 277.80 & 561 \\
\hline J155214+565916 & 16.43 & 328 & 63.16 & 328 & 49.28 & 328 \\
\hline $\mathrm{J} 164444+423304$ & 11.53 & 691 & 93.17 & 691 & 34.59 & 691 \\
\hline $\mathrm{J} 170046+622056$ & 11.11 & 455 & 49.99 & 455 & 33.32 & 455 \\
\hline $\mathrm{J} 170819+603759$ & $\ldots$ & $\ldots$ & $\ldots$ & $\ldots$ & $\ldots$ & $\ldots$ \\
\hline $\mathrm{J} 210200+000501$ & $\ldots$ & $\ldots$ & $\ldots$ & $\ldots$ & $\ldots$ & $\ldots$ \\
\hline J211343-075017 & $\ldots$ & $\ldots$ & $\ldots$ & $\ldots$ & $\ldots$ & $\ldots$ \\
\hline $\mathrm{J} 211838+005640$ & $\ldots$ & $\ldots$ & $\ldots$ & $\ldots$ & $\ldots$ & $\ldots$ \\
\hline $\mathrm{J} 212843+002435$ & $\ldots$ & $\ldots$ & $\ldots$ & $\ldots$ & $\ldots$ & $\ldots$ \\
\hline J230614-010024 & 31.65 & 344 & 202.85 & 344 & 94.96 & 344 \\
\hline J231055-090107 & $\ldots$ & $\ldots$ & $\ldots$ & $\ldots$ & $\ldots$ & $\ldots$ \\
\hline $\mathrm{J} 231317-082238$ & $\ldots$ & $\ldots$ & $\ldots$ & $\ldots$ & $\ldots$ & $\ldots$ \\
\hline $\mathrm{J} 233430+140649$ & $\ldots$ & $\ldots$ & $\ldots$ & $\ldots$ & $\ldots$ & $\ldots$ \\
\hline $\mathrm{J} 234335-005758$ & $\ldots$ & $\ldots$ & $\ldots$ & $\ldots$ & $\ldots$ & $\ldots$ \\
\hline $\mathrm{J} 234403+154214$ & 9.56 & 406 & 42.66 & 406 & 28.67 & 406 \\
\hline
\end{tabular}

Notes. The FWHM of the narrow emission lines should not be considered physical due to the low spectral resolution of our KPNO and Keck spectra.

a Scaling for the Gaussian emission lines corresponds to the integrated area under the curve as flux in units of $10^{-17} \mathrm{erg} \mathrm{s}^{-1} \mathrm{~cm}^{-2}$.

${ }^{\mathrm{b}} \mathrm{FWHM}$ is given in rest frame $\mathrm{km} \mathrm{s}^{-1}$.

While our fits do not require reddening, dust may be present along the line of sight to the AGN and/or stellar component of our PSQs. Dust reddening makes a stellar population appear less luminous and older. Therefore, if significant dust is present the true stellar population age would be younger and the mass larger than our reported measurements. For a visual extinction of $0.1 \mathrm{mag}$ a $422 \mathrm{Myr}$ population appears both older and less luminous by $\sim 10 \%$. The differences are less for older populations. The dust reddening laws toward AGNs and starbursts typically differ further complicating more detailed modeling (Calzetti et al. 1994; Richards et al. 2003).

Our selection criteria ensure that an intermediate-age population is present and strong, but we also know that a more complex stellar population is likely present that includes both older and younger stars. We know that a few of the PSQs have morphologies that show knots of ongoing star formation (C11), suggesting that young stellar populations may be present. A similarly simple model, AGN+instantaneous burst, of the prototype
PSQ, UN J1025-0040 (Brotherton et al. 1999) required revising to include a younger stellar population when high-resolution blue Hubble Space Telescope (HST) images were obtained (Brotherton et al. 2002). Because younger stellar populations have relatively weak spectral signatures and are difficult to identify with spectra alone in the presence of post-starburst populations, we want to suggest that this issue be kept in mind when interpreting spectral fitting results.

The imaging also shows galaxies that appear to be spirals with bulges, which typically have older populations, and ellipticals that appear to be post-merger remnants. The dominant intermediate age populations are most likely created as part of the merging process, but the older remain present. Older populations do have spectral signatures that are distinct (e.g., $\mathrm{Mg}$ I b absorption, larger Ca II $\mathrm{H} \& \mathrm{~K}$ ratios relative to Balmer line absorption), so we have a better chance of identifying objects that require a more complex stellar population in our fitting. $\mathrm{Mg}$ I b absorption is not readily apparent in individual spectra, 
Table 5

Starburst Properties

\begin{tabular}{|c|c|c|c|c|c|}
\hline $\begin{array}{l}\text { Object } \\
\text { SDSS }\end{array}$ & $\begin{array}{l}\text { Age } \\
(\mathrm{Myr})\end{array}$ & $\begin{array}{l}\mathrm{Raw}^{\mathrm{a}} \\
\text { Scale }\end{array}$ & $\begin{array}{c}\log \text { Mass } \\
\left(M_{\odot}\right)\end{array}$ & $\begin{array}{l}\log L_{S B}{ }^{\mathrm{b}} \\
\left(\operatorname{erg~s}^{-1}\right)\end{array}$ & $\begin{array}{l}\log L_{\mathrm{Tot}}{ }^{\mathrm{c}} \\
\left(\mathrm{erg} \mathrm{s}^{-1}\right)\end{array}$ \\
\hline J003043-103517 & 740 & 26.28 & 10.27 & 43.47 & 43.85 \\
\hline J005739+010044 & 1180 & 34.19 & 10.41 & 43.41 & 43.76 \\
\hline J015259+142738 & 1080 & 22.78 & 10.42 & 43.44 & 43.94 \\
\hline J020258-002807 & 970 & 32.25 & 10.62 & 43.67 & 43.98 \\
\hline J021447-003250 & 1090 & 22.17 & 10.54 & 43.54 & 43.98 \\
\hline J023253-082832 & 1690 & 18.80 & 10.36 & 43.15 & 43.81 \\
\hline J023700-010130 & 1230 & 36.26 & 10.79 & 43.74 & 43.92 \\
\hline J025735-001631 & 280 & 30.05 & 10.13 & 43.70 & 44.06 \\
\hline J032143-064517 & 2330 & 22.37 & 10.93 & 43.52 & 43.87 \\
\hline J040210-054630 & 2400 & 25.11 & 10.66 & 43.26 & 43.55 \\
\hline $\mathrm{J} 074621+335040$ & 620 & 48.50 & 10.41 & 43.68 & 44.04 \\
\hline $\mathrm{J} 075045+212546$ & 2330 & 25.51 & 11.11 & 43.69 & 44.39 \\
\hline $\mathrm{J} 075521+295039$ & 790 & 23.89 & 10.39 & 43.54 & 43.83 \\
\hline J075549+321704 & 2310 & 21.06 & 11.06 & 43.64 & 44.14 \\
\hline J081018+250921 & 90 & 62.48 & 9.58 & 43.58 & 44.28 \\
\hline $\mathrm{J} 105816+102414$ & 1180 & 51.56 & 10.68 & 43.67 & 43.96 \\
\hline J115159+673604 & 1490 & 31.75 & 10.57 & 43.44 & 43.63 \\
\hline $\mathrm{J} 115355+582442$ & 760 & 38.21 & 10.52 & 43.71 & 43.97 \\
\hline $\mathrm{J} 123043+614821$ & 1340 & 21.92 & 10.54 & 43.46 & 43.92 \\
\hline $\mathrm{J} 124833+563507$ & 1400 & 67.37 & 10.83 & 43.75 & 43.97 \\
\hline $\mathrm{J} 140513+625008$ & 830 & 34.36 & 10.73 & 43.85 & 44.05 \\
\hline $\mathrm{J} 145640+524727$ & 1890 & 21.89 & 10.52 & 43.25 & 43.66 \\
\hline $\mathrm{J} 145658+593202$ & 960 & 17.13 & 10.30 & 43.36 & 43.67 \\
\hline $\mathrm{J} 154534+573625$ & 2380 & 44.53 & 10.89 & 43.51 & 43.95 \\
\hline $\mathrm{J} 155214+565916$ & 1210 & 16.23 & 10.41 & 43.37 & 43.81 \\
\hline $\mathrm{J} 164444+423304$ & 470 & 23.92 & 10.11 & 43.49 & 43.83 \\
\hline $\mathrm{J} 170046+622056$ & 2670 & 28.28 & 10.78 & 43.34 & 43.66 \\
\hline $\mathrm{J} 170819+603759$ & 1270 & 20.73 & 10.37 & 43.32 & 43.68 \\
\hline $\mathrm{J} 210200+000501$ & 790 & 54.88 & 10.73 & 43.89 & 44.11 \\
\hline $\mathrm{J} 211343-075017$ & 750 & 52.35 & 10.97 & 44.12 & 44.34 \\
\hline $\mathrm{J} 211838+005640$ & 1160 & 41.20 & 10.95 & 43.91 & 44.18 \\
\hline $\mathrm{J} 212843+002435$ & 1690 & 18.88 & 10.65 & 43.42 & 43.88 \\
\hline $\mathrm{J} 230614-010024$ & 890 & 48.31 & 10.50 & 43.62 & 43.95 \\
\hline $\mathrm{J} 231055-090107$ & 2230 & 31.48 & 11.05 & 43.67 & 43.90 \\
\hline $\mathrm{J} 231317-082238$ & 1500 & 28.73 & 10.85 & 43.69 & 44.07 \\
\hline $\mathrm{J} 233430+140649$ & 240 & 18.19 & 9.84 & 43.49 & 44.12 \\
\hline $\mathrm{J} 234335-005758$ & 1400 & 36.17 & 10.84 & 43.73 & 43.95 \\
\hline $\mathrm{J} 234403+154214$ & 2570 & 34.88 & 10.90 & 43.47 & 43.64 \\
\hline
\end{tabular}

Notes.

a Scale directly from fitting.

b Total integrated light of the starburst component from $3000 \AA$ to $6000 \AA$.

c Total integrated light (AGNs plus starburst components) from $3000 \AA$ to $6000 \AA$ A.

but we note that it is visible in composite PSQ spectra we have examined.

We performed an additional set of fits in which we also included an older stellar population with an age of 5.6 Gyr. This additional component did not result in improved fits (as evaluated by $\chi^{2}$ and examining by eye the absorption lines in detail). This could be the result of degeneracies inherent in fitting many components to a complex spectrum, something worth additional study, or because the older components do not contribute significantly to the observed flux. This does not mean that they are not present, only that the fits may be degenerate, or that the older components are weak. Others have investigated the issues involving fitting multiple-aged stellar components to a spectrum with a single-aged stellar synthesis model, e.g., Serra \& Trager 2007; Trager \& Somerville 2009, who have characterized some of the resulting biases and limitations.
We quantitatively investigated this effect ourselves with our own techniques by simulating spectra of a post-starburst plus old stellar component for a range of flux/mass ratios. We created our simulated spectra by combining ratios of $422 \mathrm{Myr}$ old and 5.6 Gyr old Charlot \& Bruzual instantaneous burst models and adding artificial noise consistent with our data. We then fit the resulting simulated spectra with a single-aged stellar component. The intermediate stellar age and mass were recovered to better than $10 \%$ as long as the older population was less than $70 \%$ of the stellar mass. At higher mass fractions, the intermediate age population fit was compromised and skewed the results to indicate older, more massive populations. The AGN component additionally greatly complicates the task and can mask the presence of different-aged stellar components. We conclude that our results are robust as long as the post-starburst population is massive enough to be dominant in flux, as it is at least in some cases (e.g., Hiner et al. 2012), but that higher S/N spectra (letting us measure the $\mathrm{Mg}$ I $\mathrm{b}$ feature more reliably) or another waveband of high-resolution imaging (e.g., Brotherton et al. 2002) will be required to definitively resolve this issue.

We additionally note that in our simulations, when a fit overestimates a post-starburst stellar population age, the equivalent widths of the Balmer lines are greatly overestimated (at least $\sim 30 \%$ ), although as a complication the AGN component can dilute the equivalent widths. In our single intermediateage population fits, the Balmer lines of the data are well characterized by our model and for only one object (SDSS J023700.30-010130.5) we note that there might be a significant overestimation. The good fits overall and the fact that we have used a single, consistent approach to fitting all objects suggests that even in the presence of some systematic issues, relative measurements are still likely meaningful and correlation analysis is of interest.

The issue of how to robustly fit complex spectra involving multiple stellar components of unknown metallicity, an AGN, and unknown and perhaps complex dust reddening, at a range of $\mathrm{S} / \mathrm{Ns}$, is complicated and deserving of additional deeper investigation and is beyond the scope of the present work. Our existing data are well and consistently fit by our simple model, and to investigate these more sophisticated models would require additional data. Even with more data, degeneracies may make it extremely difficult to find unique and perfect solutions. Again, our fits appear good and likely provide useful information about the PSQs, but keep in mind these caveats and how they may bias the results when interpreting our measurements.

\subsection{AGN Properties}

From the fitting results, we were able to estimate two fundamental physical AGN properties: the $\mathrm{BH}$ mass and Eddington ratio. We employed scaling relations that extend the reverberation mapping results of physical properties (i.e., $M_{\mathrm{BH}}$ and radius of the broad-line region) to the observable quantities (i.e., continuum luminosity and broad line FWHM) of single-epoch data to calculate $\mathrm{BH}$ mass. We used $\mathrm{Mg}$ II, $\mathrm{H} \beta, \mathrm{H} \alpha$ as well as estimated values of $\mathrm{H} \beta$ based on the measurements of $\mathrm{H} \alpha$, being careful to discard data of low quality. All of our spectra have coverage at $5100 \AA$. We used the $5100 \AA$ monochromatic luminosities based on the power-law components used in the fits as our AGN luminosities for the scaling relations below.

From the relation given by Vestergaard \& Osmer (2009), we estimated $M_{\mathrm{BH}}$ based on the Mg II broad line FWHM and the 
$5100 \AA$ Å monochromatic luminosity:

$$
\frac{M_{\mathrm{BH}}(\mathrm{Mg} \mathrm{II})}{M_{\odot}}=10^{6.96}\left[\frac{\mathrm{FWHM} \mathrm{Mg} \mathrm{II}}{1000 \mathrm{~km} \mathrm{~s}^{-1}}\right]^{2}\left[\frac{\lambda L_{\lambda}(5100 \AA)}{10^{44} \mathrm{erg} \mathrm{s}^{-1}}\right]^{0.5} .
$$

The scatter in the zero point of the relation is given to be 0.55 dex.

We used the relation

$$
\frac{M_{\mathrm{BH}}(\mathrm{H} \beta)}{M_{\odot}}=10^{6.91 \pm 0.02}\left[\frac{\mathrm{FWHM} \mathrm{H} \beta}{1000 \mathrm{~km} \mathrm{~s}^{-1}}\right]^{2}\left[\frac{\lambda L_{\lambda}(5100 \AA)}{10^{44} \mathrm{erg} \mathrm{s}^{-1}}\right]^{0.5}
$$

to calculate the $\mathrm{BH}$ mass based on the $\mathrm{H} \beta$ broad line FWHM and the $5100 \AA$ AGN monochromatic luminosity (Vestergaard \& Peterson 2006). The intrinsic scatter in the sample is described by 0.43 dex. We have given the relation in linear space as opposed to $\log$ space for ease of reading. We note that the relations given for $\mathrm{Mg}$ II and $\mathrm{H} \beta$ are on the same mass scale.

We estimated the $\mathrm{BH}$ masses using $\mathrm{H} \alpha$ broad line FWHM and the $5100 \AA$ AGN monochromatic luminosity according to the Greene et al. (2010) scaling relation:

$$
\begin{aligned}
\frac{M_{\mathrm{BH}}(\mathrm{H} \alpha)}{M_{\odot}}= & (9.7 \pm 0.5) \times 10^{6}\left[\frac{\mathrm{FWHM} \mathrm{H} \alpha}{1000 \mathrm{~km} \mathrm{~s}^{-1}}\right]^{2.06 \pm 0.06} \\
& \times\left[\frac{\lambda L_{\lambda}(5100 \AA)}{10^{44} \mathrm{erg} \mathrm{s}^{-1}}\right]^{0.519 \pm 0.07} \cdot
\end{aligned}
$$

Though the scatter in this relation is not reported, typical values are $\sim 0.4-0.5$ dex.

Since stellar absorption from the post-starburst population contaminates broad emission in $\mathrm{H} \beta$, making the fits less reliable, and this stellar contamination is negligible for $\mathrm{H} \alpha$ we used $\mathrm{H} \alpha$ as a proxy for $\mathrm{H} \beta$ when $\mathrm{H} \alpha$ is present in the spectrum (Shen et al. 2011)

$$
\begin{aligned}
\log \left(\frac{\mathrm{FWHM} \mathrm{H} \beta}{\mathrm{km} \mathrm{s}^{-1}}\right)= & (-0.11 \pm 0.03) \\
& +(1.05 \pm 0.01) \times \log \left(\frac{\mathrm{FWHM} \mathrm{H} \alpha}{\mathrm{km} \mathrm{s}^{-1}}\right) .
\end{aligned}
$$

We then used the estimated $\mathrm{H} \beta$ values in conjunction with Equation (2) to calculate $\mathrm{BH}$ measurements for $\mathrm{H} \beta$ based on $\mathrm{H} \alpha$. There are slight differences between the two formalisms of Greene et al. (2010) and Vestergaard \& Peterson (2006); most notably between the prefactors and radius-luminosity (scaling of $\lambda L_{\lambda}$ ) relations. However, the differences are small; for our sample the difference is less than $8 \%$, much less than the intrinsic scatter.

We have given several measurements of the BH mass. Each line has its own particular issue. The blue side of the spectra suffer from $\mathrm{S} / \mathrm{N}$ degradation which sometimes gives unreliable measurements for $\mathrm{Mg}$ II. Occasionally, up to $\sim 50 \%$ of the $\mathrm{H} \beta$ broad emission can be contaminated by stellar absorption. Due to the redshift range of our sample, sometimes $\mathrm{H} \alpha$ is out of our observing window. In subsequent sections, we make comparisons using these values individually. However, we also give an adopted $M_{\mathrm{BH}}$ by applying the following prescription. When we have three reliable measurements we adopt the median of these values. For two good measurements, we give the mean

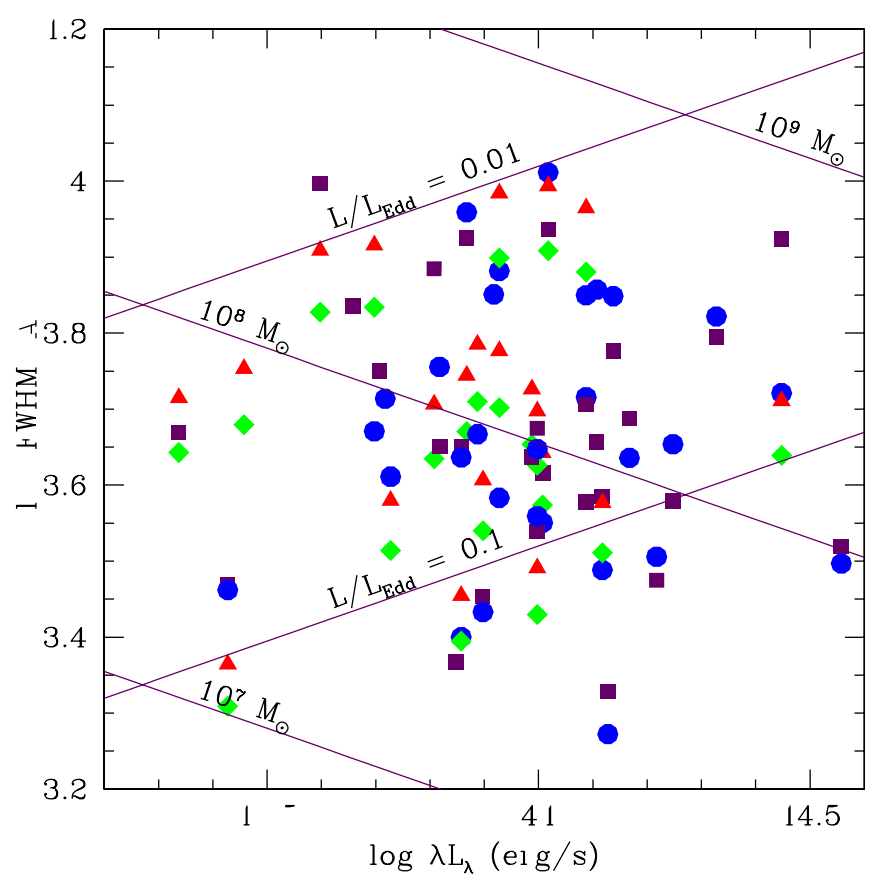

Figure 2. FWHM vs. $\lambda L_{\lambda}(5100 \AA)$ based on measurements from $\mathrm{Mg}$ II (purple squares), $\mathrm{H} \beta$ (blue circles), $\mathrm{H} \alpha$ (green diamonds), and $\mathrm{H} \beta$ predicted from $\mathrm{H} \alpha$ (red triangles). Lines of constant $M_{\mathrm{BH}}$ and $L / L_{\mathrm{Edd}}$ are shown for Mg II. PSQs have black hole masses of $\sim 10^{8} M_{\odot}$ and Eddington fractions of several percent. (A color version of this figure is available in the online journal.)

of the values. If there is only one reliable value, we adopt this as our $M_{\mathrm{BH}}$. There is one object (SDSS J020258.94-002807.5) for which $\mathrm{H} \alpha$ was not covered and both $\mathrm{Mg}$ II and $\mathrm{H} \beta$ were unreliable.

We estimated the AGN bolometric luminosity using the measured rest-frame flux at $5100 \AA$ from the fit to the powerlaw continuum and convert it into a total emitted luminosity using luminosity distances for our cosmology and an appropriate bolometric correction ( $f=8.1$; Runnoe et al. 2012):

$$
L_{\mathrm{bol}}=4 \pi D_{L}^{2} f(1+z) \lambda F_{\lambda}(5100 \AA)
$$

The Eddington ratio is given by $L_{\mathrm{bol}} / L_{\mathrm{Edd}}$ where we use $L_{\mathrm{Edd}}=$ $1.51 \times 10^{38}\left(M_{\mathrm{BH}} / M_{\odot}\right) \mathrm{erg} \mathrm{s}^{-1}$ (Krolik 1999). Table 6 gives BH masses for each broad line measurement and their Eddington ratios.

Figure 2 shows where the different broad line width measurements (i.e., $\mathrm{Mg}$ II, $\mathrm{H} \beta, \mathrm{H} \alpha$, and predicted $\mathrm{H} \beta$ ) lie as a function of $\lambda L_{\lambda}(5100 \AA)$. For reference we indicate lines of constant $M_{\mathrm{BH}}$ and $L_{\mathrm{bol}} / L_{\mathrm{Edd}}$ based on Mg II. Constant lines of $M_{\mathrm{BH}}$ and $L_{\text {bol }} / L_{\text {Edd }}$ based on $\mathrm{H} \beta$ and $\mathrm{H} \alpha$ will vary slightly in slope and intercept.

\subsection{Post-starburst Properties}

The post-starburst stellar populations can be described using two fundamental physical parameters, starburst age and mass. The post-starburst ages are known directly from the fitting results. The scale factor is a function of the mass and age of the starburst. The inputted PSQ spectra are in units of $10^{-17} \mathrm{erg} \mathrm{s}^{-1} \mathrm{~cm}^{2} \AA^{-1}$ while the template spectra are in units of $L_{\odot} / \AA$ and scaled up by their age in Myr. Thus, we can derive the total starburst mass by using the scale factor and the 
Table 6

AGN Properties

\begin{tabular}{|c|c|c|c|c|c|c|c|c|c|c|c|c|}
\hline \multirow{2}{*}{$\begin{array}{l}\text { Object } \\
\text { SDSS }\end{array}$} & \multicolumn{5}{|c|}{$\log M_{\mathrm{BH}}\left(M_{\odot}\right)$} & \multicolumn{5}{|c|}{$L / L_{\text {Edd }}$} & \multirow{2}{*}{$f_{\text {nuc }}{ }^{\mathrm{a}}$} & \multirow{2}{*}{$\begin{array}{c}\log L_{\mathrm{AGN}} \mathrm{b} \\
\left(\mathrm{erg} \mathrm{s}^{-1}\right)\end{array}$} \\
\hline & Mg II & $\mathrm{H} \beta$ & $\mathrm{H} \alpha$ & $\mathrm{H} \alpha \rightarrow \mathrm{H} \beta$ & Adopted & $\mathrm{Mg}_{\text {II }}$ & $\mathrm{H} \beta$ & $\mathrm{H} \alpha$ & $\mathrm{H} \alpha \rightarrow \mathrm{H} \beta$ & Adopted & & \\
\hline J003043-103517 & $\ldots$ & 8.64 & 8.80 & 8.84 & 8.73 & $\ldots$ & 0.011 & 0.007 & 0.007 & 0.009 & 0.581 & 43.61 \\
\hline J005739+010044 & 8.36 & $\ldots$ & 8.19 & 8.23 & 8.29 & 0.015 & $\ldots$ & 0.022 & 0.020 & 0.018 & 0.552 & 43.50 \\
\hline J015259+142738 & 7.93 & 8.02 & 8.17 & 8.20 & 8.02 & 0.065 & 0.053 & 0.037 & 0.035 & 0.053 & 0.687 & 43.78 \\
\hline J020258-002807 & $\ldots$ & $\ldots$ & $\ldots$ & $\ldots$ & $\ldots$ & $\ldots$ & $\ldots$ & $\ldots$ & $\ldots$ & $\ldots$ & 0.502 & 43.68 \\
\hline J021447-003250 & 7.89 & 8.39 & $\ldots$ & $\ldots$ & 8.21 & 0.085 & 0.027 & $\ldots$ & $\ldots$ & 0.041 & 0.640 & 43.79 \\
\hline J023253-082832 & 8.15 & 8.65 & 8.85 & 8.88 & 8.65 & 0.047 & 0.015 & 0.009 & 0.009 & 0.015 & 0.778 & 43.70 \\
\hline J023700-010130 & 8.19 & $\ldots$ & $\ldots$ & $\ldots$ & 8.19 & 0.016 & $\ldots$ & $\ldots$ & $\ldots$ & 0.016 & 0.342 & 43.46 \\
\hline J025735-001631 & 7.41 & 7.52 & $\ldots$ & $\ldots$ & 7.47 & 0.278 & 0.218 & $\ldots$ & $\ldots$ & 0.244 & 0.567 & 43.82 \\
\hline J032143-064517 & 7.90 & 8.33 & $\ldots$ & $\ldots$ & 8.17 & 0.044 & 0.016 & $\ldots$ & $\ldots$ & 0.024 & 0.552 & 43.61 \\
\hline J040210-054630 & 7.34 & 7.55 & 7.33 & 7.35 & 7.34 & 0.065 & 0.041 & 0.068 & 0.064 & 0.065 & 0.484 & 43.24 \\
\hline J074621+335040 & 7.92 & 7.95 & 8.10 & 8.12 & 7.95 & 0.085 & 0.080 & 0.056 & 0.053 & 0.080 & 0.563 & 43.79 \\
\hline J075045+212546 & 8.01 & 8.18 & $\ldots$ & $\ldots$ & 8.11 & 0.192 & 0.128 & $\ldots$ & $\ldots$ & 0.153 & 0.800 & 44.29 \\
\hline J075521+295039 & 7.35 & $\ldots$ & $\ldots$ & $\ldots$ & 7.35 & 0.169 & $\ldots$ & $\ldots$ & $\ldots$ & 0.169 & 0.487 & 43.52 \\
\hline J075549+321704 & 7.97 & 8.34 & $\ldots$ & $\ldots$ & 8.19 & 0.101 & 0.043 & $\ldots$ & $\ldots$ & 0.060 & 0.685 & 43.97 \\
\hline J081018+250921 & 8.76 & 8.57 & 8.54 & 8.56 & 8.57 & 0.026 & 0.040 & 0.044 & 0.042 & 0.040 & 0.801 & 44.19 \\
\hline J105816+102414 & 8.48 & 8.76 & 8.30 & 8.33 & 8.48 & 0.013 & 0.007 & 0.020 & 0.018 & 0.013 & 0.482 & 43.64 \\
\hline $\mathrm{J} 115159+673604$ & $\ldots$ & $\ldots$ & 8.11 & 8.15 & 8.11 & $\ldots$ & $\ldots$ & 0.012 & 0.011 & 0.012 & 0.359 & 43.19 \\
\hline $\mathrm{J} 115355+582442$ & 7.55 & 7.73 & 8.05 & 8.07 & 7.73 & 0.121 & 0.080 & 0.038 & 0.036 & 0.080 & 0.460 & 43.63 \\
\hline $\mathrm{J} 123043+614821$ & 7.77 & 8.03 & 8.27 & 8.30 & 8.03 & 0.091 & 0.050 & 0.028 & 0.027 & 0.050 & 0.649 & 43.73 \\
\hline $\mathrm{J} 124833+563507$ & $\ldots$ & 8.19 & 8.39 & 8.43 & 8.30 & $\ldots$ & 0.027 & 0.017 & 0.016 & 0.021 & 0.403 & 43.58 \\
\hline $\mathrm{J} 140513+625008$ & 7.96 & $\ldots$ & 8.33 & 8.36 & 8.18 & 0.057 & $\ldots$ & 0.025 & 0.023 & 0.034 & 0.377 & 43.63 \\
\hline $\mathrm{J} 145640+524727$ & $\ldots$ & 8.00 & 7.90 & 7.93 & 7.95 & $\ldots$ & 0.029 & 0.036 & 0.033 & 0.032 & 0.609 & 43.44 \\
\hline J145658+593202 & $\ldots$ & 8.10 & 8.55 & 8.59 & 8.38 & $\ldots$ & 0.021 & 0.008 & 0.007 & 0.011 & 0.513 & 43.38 \\
\hline $\mathrm{J} 154534+573625$ & 8.57 & 8.94 & 8.86 & 8.90 & 8.86 & 0.015 & 0.006 & 0.008 & 0.007 & 0.008 & 0.644 & 43.76 \\
\hline J155214+565916 & $\ldots$ & 8.04 & 8.40 & 8.43 & 8.25 & $\ldots$ & 0.042 & 0.018 & 0.017 & 0.025 & 0.643 & 43.62 \\
\hline J164444+423304 & 7.41 & 7.64 & 7.72 & 7.75 & 7.64 & 0.148 & 0.088 & 0.072 & 0.069 & 0.088 & 0.541 & 43.56 \\
\hline $\mathrm{J} 170046+622056$ & 8.48 & $\ldots$ & 8.48 & 8.53 & 8.48 & 0.007 & $\ldots$ & 0.007 & 0.006 & 0.007 & 0.528 & 43.39 \\
\hline J170819+603759 & $\ldots$ & 8.20 & $\ldots$ & $\ldots$ & 8.20 & $\ldots$ & 0.018 & $\ldots$ & $\ldots$ & 0.018 & 0.556 & 43.42 \\
\hline $\mathrm{J} 210200+000501$ & 7.92 & 8.11 & $\ldots$ & $\ldots$ & 8.03 & 0.046 & 0.030 & $\ldots$ & $\ldots$ & 0.036 & 0.391 & 43.70 \\
\hline $\mathrm{J} 211343-075017$ & 8.31 & 8.68 & $\ldots$ & $\ldots$ & 8.53 & 0.036 & 0.016 & $\ldots$ & $\ldots$ & 0.022 & 0.394 & 43.94 \\
\hline $\mathrm{J} 211838+005640$ & 8.06 & 8.68 & $\ldots$ & $\ldots$ & 8.47 & 0.060 & 0.014 & $\ldots$ & $\ldots$ & 0.023 & 0.469 & 43.85 \\
\hline $\mathrm{J} 212843+002435$ & 7.75 & 8.03 & $\ldots$ & $\ldots$ & 7.91 & 0.158 & 0.082 & $\ldots$ & $\ldots$ & 0.108 & 0.652 & 43.69 \\
\hline $\mathrm{J} 230614-010024$ & 8.04 & 8.20 & 7.87 & 7.89 & 8.04 & 0.049 & 0.033 & 0.072 & 0.068 & 0.049 & 0.535 & 43.68 \\
\hline $\mathrm{J} 231055-090107$ & 8.05 & $\ldots$ & $\ldots$ & $\ldots$ & 8.05 & 0.025 & $\ldots$ & $\ldots$ & $\ldots$ & 0.025 & 0.413 & 43.51 \\
\hline $\mathrm{J} 231317-082238$ & 8.15 & 8.27 & $\ldots$ & $\ldots$ & 8.21 & 0.056 & 0.043 & $\ldots$ & $\ldots$ & 0.049 & 0.591 & 43.84 \\
\hline $\mathrm{J} 233430+140649$ & 8.45 & 8.72 & $\ldots$ & $\ldots$ & 8.60 & 0.041 & 0.022 & $\ldots$ & $\ldots$ & 0.029 & 0.764 & 44.00 \\
\hline $\mathrm{J} 234335-005758$ & $\ldots$ & 8.57 & $\ldots$ & $\ldots$ & 8.57 & $\ldots$ & 0.012 & $\ldots$ & $\ldots$ & 0.012 & 0.406 & 43.56 \\
\hline $\mathrm{J} 234403+154214$ & 7.70 & 9.18 & 7.97 & 8.01 & 7.97 & 0.023 & 0.001 & 0.013 & 0.011 & 0.013 & 0.326 & 43.15 \\
\hline
\end{tabular}

Notes.

a Integrated ratio of AGNs to total light from $3000 \AA$ to $6000 \AA$.

b Total integrated light of the AGN power law from $3000 \AA$ to $6000 \AA$.

outputted age:

$$
\begin{aligned}
\frac{M_{\mathrm{SB}}}{M_{\odot}}= & \text { Raw scale } \times \text { Age }(\mathrm{Myr}) \times 10^{-17} \mathrm{erg} \mathrm{s}^{-1} \mathrm{~cm}^{2} \AA^{-1} \\
& \times \frac{3.826 \times 10^{33} \mathrm{erg} \mathrm{s}^{-1} \AA^{-1}}{4 \pi D_{L}^{2}(1+z)} .
\end{aligned}
$$

The expression in brackets is the solar flux at the luminosity distance, $D_{L}$. Thus, the ratio between the scaled model and the solar flux at $D_{L}$ gives the starburst mass. Table 5 gives the starburst fitting results, derived starburst masses, and integrated luminosities.

\section{RESULTS AND DISCUSSION}

We seek to explore joint AGNs and starburst activity by investigating the interplay between the fundamental properties of PSQs and their morphological subpopulations. In
Section 4.1, we investigate the relationships between the fundamental properties of PSQs. We investigate the fundamental properties of PSQs in relation to their morphological subpopulations in Section 4.2. Finally, in Section 4.3, we give a simple prescription to reliably classify the morphology of PSQs based on spectral properties.

\subsection{Correlations Based on Fundamental PSQ Properties}

We calculate Spearman-rank correlation coefficient matrices involving several hundred correlation tests between fitted and derived parameters for (1) the total sample, (2) the early-type and spiral morphological classifications, and (3) the disturbed and undisturbed classifications. We compute the probability of the correlations arising by chance and list those less than $1 \%$ in Table 7.

We find a number of strong correlations among quasar and starburst properties; however, many of these are due to selection effects which we will discuss below. Some merger 
Table 7

Significant Correlations

\begin{tabular}{|c|c|c|c|c|}
\hline Property 1 & Property 2 & $\rho$ & $P(\%)$ & Number \\
\hline \multicolumn{5}{|c|}{ Total Population } \\
\hline$z$ & $L_{\text {Tot }}$ & 0.518 & 0.086 & 38 \\
\hline$z$ & $L_{S B}$ & 0.494 & 0.161 & 38 \\
\hline$z$ & SB Mass & 0.428 & 0.737 & 38 \\
\hline$\lambda L_{\lambda}(5100 \AA)^{\mathrm{a}}$ & $L / L_{\text {Edd }}$ Adopted & 0.433 & 0.740 & 37 \\
\hline$\lambda L_{\lambda}(5100 \AA)$ & {$[\mathrm{O} \mathrm{III}] / \mathrm{H} \beta$} & 0.481 & 0.527 & 32 \\
\hline$\lambda L_{\lambda}(5100 \AA)$ & {$[\mathrm{O} \mathrm{III}] /[\mathrm{O} \mathrm{II}]$} & 0.476 & 0.508 & 33 \\
\hline$\alpha$ & SB Mass & 0.479 & 0.237 & 38 \\
\hline$L_{\text {Tot }}$ & {$[\mathrm{O} \mathrm{III}] / \mathrm{H} \beta$} & 0.509 & 0.292 & 32 \\
\hline$L_{\text {Tot }}$ & {$[\mathrm{N}$ II $] / \mathrm{H} \alpha$} & 0.678 & 0.073 & 21 \\
\hline$L_{\mathrm{AGN}}$ & $L_{\mathrm{SB}}$ & 0.441 & 0.553 & 38 \\
\hline$L_{\mathrm{AGN}}$ & $L / L_{\text {Edd }}$ Adopted & 0.424 & 0.890 & 37 \\
\hline$L_{\mathrm{AGN}}$ & {$[\mathrm{O}$ III $] / \mathrm{H} \beta$} & 0.563 & 0.079 & 32 \\
\hline$L_{\mathrm{AGN}}$ & {$[\mathrm{O} \mathrm{III}] /[\mathrm{O} \mathrm{II}]$} & 0.493 & 0.359 & 33 \\
\hline$L_{\mathrm{SB}}$ & SB Mass & 0.452 & 0.438 & 38 \\
\hline$L_{\mathrm{SB}}$ & {$[\mathrm{N}$ II $] / \mathrm{H} \alpha$} & 0.549 & 0.990 & 21 \\
\hline$L_{\mathrm{SB}}$ & {$[\mathrm{Ne} \mathrm{v}] /[\mathrm{Ne} \mathrm{III}]$} & 0.504 & 0.276 & 33 \\
\hline$M_{\mathrm{BH}} \mathrm{Mg}$ II & $L / L_{\text {Edd }} M g$ II & -0.742 & 0.000 & 29 \\
\hline$M_{\mathrm{BH}} \mathrm{H} \beta$ & $L / L_{\text {Edd }} \mathrm{H} \beta$ & -0.809 & 0.000 & 30 \\
\hline$M_{\mathrm{BH}} \mathrm{H} \alpha$ & $L / L_{\text {Edd }} \mathrm{H} \alpha$ & -0.739 & 0.013 & 21 \\
\hline$M_{\mathrm{BH}} \mathrm{H} \alpha-\mathrm{H} \beta$ & $L / L_{\text {Edd }} \mathrm{H} \alpha-\mathrm{H} \beta$ & -0.739 & 0.013 & 21 \\
\hline$M_{\mathrm{BH}}$ Adopted & $L / L_{\text {Edd }}$ Adopted & -0.724 & 0.000 & 37 \\
\hline SB Mass & SB Age & 0.631 & 0.002 & 38 \\
\hline$[\mathrm{O} \mathrm{III}] / \mathrm{H} \beta$ & {$[\mathrm{N} \mathrm{II}] / \mathrm{H} \alpha$} & 0.838 & 0.003 & 17 \\
\hline$[\mathrm{O} \mathrm{III}] / \mathrm{H} \beta$ & {$[\mathrm{O} \mathrm{III}] /\left[\mathrm{O}_{\mathrm{II}}\right]$} & 0.737 & 0.000 & 31 \\
\hline$[\mathrm{N}$ II $] / \mathrm{H} \alpha$ & {$[\mathrm{O} \mathrm{III}] /[\mathrm{O} \mathrm{II}]$} & 0.757 & 0.043 & 17 \\
\hline \multicolumn{5}{|c|}{ Elliptical Population } \\
\hline$M_{\mathrm{BH}} \mathrm{Mg}$ II & $L / L_{\text {Edd }} M g$ II & -0.790 & 0.222 & 12 \\
\hline$M_{\mathrm{BH}} \mathrm{H} \beta$ & $L / L_{\text {Edd }} \mathrm{H} \beta$ & -0.841 & 0.032 & 13 \\
\hline$M_{\mathrm{BH}}$ Adopted & $L / L_{\text {Edd }}$ Adopted & -0.692 & 0.873 & 13 \\
\hline$\underline{\text { SB Mass }}$ & SB Age & 0.747 & 0.333 & 13 \\
\hline \multicolumn{5}{|c|}{ Spiral Population } \\
\hline$\alpha$ & SB Mass & 0.824 & 0.053 & 13 \\
\hline$\alpha$ & SB Age & 0.764 & 0.238 & 13 \\
\hline$M_{\mathrm{BH}}$ Adopted & $L / L_{\text {Edd }}$ Adopted & -0.753 & 0.298 & 13 \\
\hline SB Mass & SB Age & 0.813 & 0.072 & 13 \\
\hline \multicolumn{5}{|c|}{ Undisturbed Population } \\
\hline$\alpha$ & SB Mass & 0.824 & 0.005 & 17 \\
\hline$L_{\text {Tot }}$ & {$[\mathrm{O} \mathrm{III}] /[\mathrm{O} \mathrm{II}]$} & 0.650 & 0.871 & 15 \\
\hline$L_{\mathrm{AGN}}$ & $L_{\mathrm{SB}}$ & 0.674 & 0.301 & 17 \\
\hline$L_{\mathrm{AGN}}$ & {$[\mathrm{O} \mathrm{III}] /[\mathrm{O} \mathrm{II}]$} & 0.657 & 0.777 & 15 \\
\hline$M_{\mathrm{BH}} \mathrm{Mg}$ II & $L / L_{\text {Edd }} M g$ II & -0.829 & 0.024 & 14 \\
\hline$M_{\mathrm{BH}} \mathrm{H} \beta$ & $L / L_{\text {Edd }} \mathrm{H} \beta$ & -0.934 & 0.000 & 14 \\
\hline$M_{\mathrm{BH}} \mathrm{H} \alpha$ & $L / L_{\text {Edd }} \mathrm{H} \alpha$ & -0.800 & 0.311 & 11 \\
\hline$M_{\mathrm{BH}} \mathrm{H} \alpha-\mathrm{H} \beta$ & $L / L_{\text {Edd }} \mathrm{H} \alpha-\mathrm{H} \beta$ & -0.800 & 0.311 & 11 \\
\hline$M_{\mathrm{BH}}$ Adopted & $L / L_{\text {Edd }}$ Adopted & -0.814 & 0.007 & 17 \\
\hline SB Mass & SB Age & 0.620 & 0.792 & 17 \\
\hline SB Mass & {$[\mathrm{O} \mathrm{III}] /[\mathrm{O} \mathrm{II}]$} & 0.646 & 0.921 & 15 \\
\hline$[\mathrm{O} \mathrm{III}] / \mathrm{H} \beta$ & [O III $] /[\mathrm{O} \mathrm{II]}$ & 0.842 & 0.016 & 14 \\
\hline
\end{tabular}

Note. a $\lambda L_{\lambda}(5100 \AA)$ is the monochromatic AGN luminosity at $5100 \AA$ in $\operatorname{erg~s}^{-1}$.

induced evolutionary scenarios predict correlations among these properties of a more physical origin (Di Matteo et al. 2005). We find very few additional correlations of this type.

Our selection criteria provides an efficient means for selecting the brightest systems with both AGNs and post-starburst populations. However, this then creates some artificial correlations between parameters. For example, the starburst and AGN must be comparable in luminosity in order for one not to swamp out

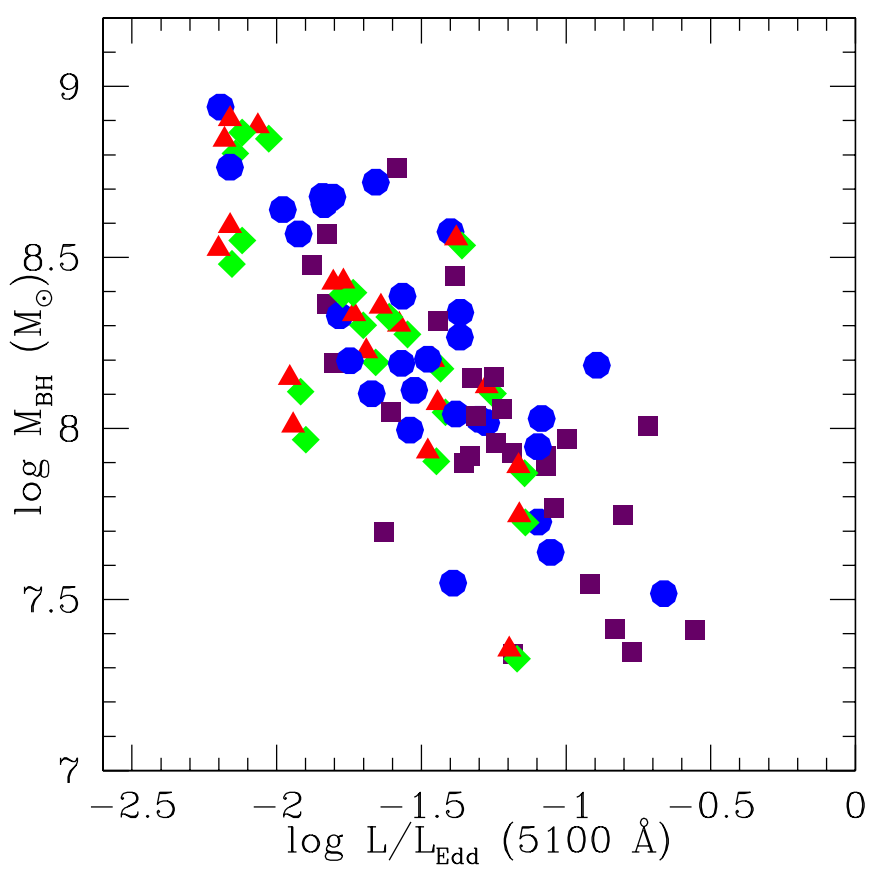

Figure 3. Relation between $M_{\mathrm{BH}}$ and $L / L_{\mathrm{Edd}}(5100 \AA)$ based on measurements from $\mathrm{Mg}$ II (purple squares), $\mathrm{H} \beta$ (blue circles), $\mathrm{H} \alpha$ (green diamonds), and $\mathrm{H} \beta$ predicted from $\mathrm{H} \alpha$ (red triangles). The inverse correlation is likely the result of two effects: cosmic downsizing and our luminosity cut.

(A color version of this figure is available in the online journal.)

light from the other, thus giving rise to a significant correlation of the luminosities of both. We note that the mean AGN-to-total light, $f_{\text {nuc }}$, is 0.55 with only a standard deviation of 0.13 . This leaves us with a limited parameter space to explore.

\subsubsection{Derived AGN Properties}

Figure 3 shows our strongest correlation among AGN properties. The $M_{\mathrm{BH}}$ increases as $L / L_{\mathrm{Edd}}$ decreases, which probably arises as a result of two effects. The first effect is the dearth in massive BHs with high accretion rates at this redshift, which is the result of cosmic downsizing (Heckman et al. 2004). The second cause is the lack of lower mass black holes with low accretion rates which fail to make the luminosity cut. The combination of these two effects, demographics plus selection effects, leads to a strong inverse correlation. However, this does not rule out the possibility that there is an underlying physical correlation, but confirming this would require a more sophisticated sample selection.

\subsubsection{Narrow-line Ratios}

Narrow emission lines can be powered by several sources of photoionizing radiation, including young $\mathrm{O}$ and $\mathrm{B}$ stars and the central AGN. Comparing the relative strengths between lines with high- and low-ionization potentials tells us about the shape of the photoionizing continuum and thus diagnoses the source. AGNs have harder continua (relatively more high-energy photons) while star-forming continua are softer (relatively more low-energy photons).

We find a number of strong correlations among narrow-line ratios that can be interpreted in terms of the relative contributions of AGNs and star formation. In particular, the highly ionized [O III] $\lambda 5007$ emission line is more prominent in AGN spectra but other lines may also be diagnostic. For example, Figure 4 


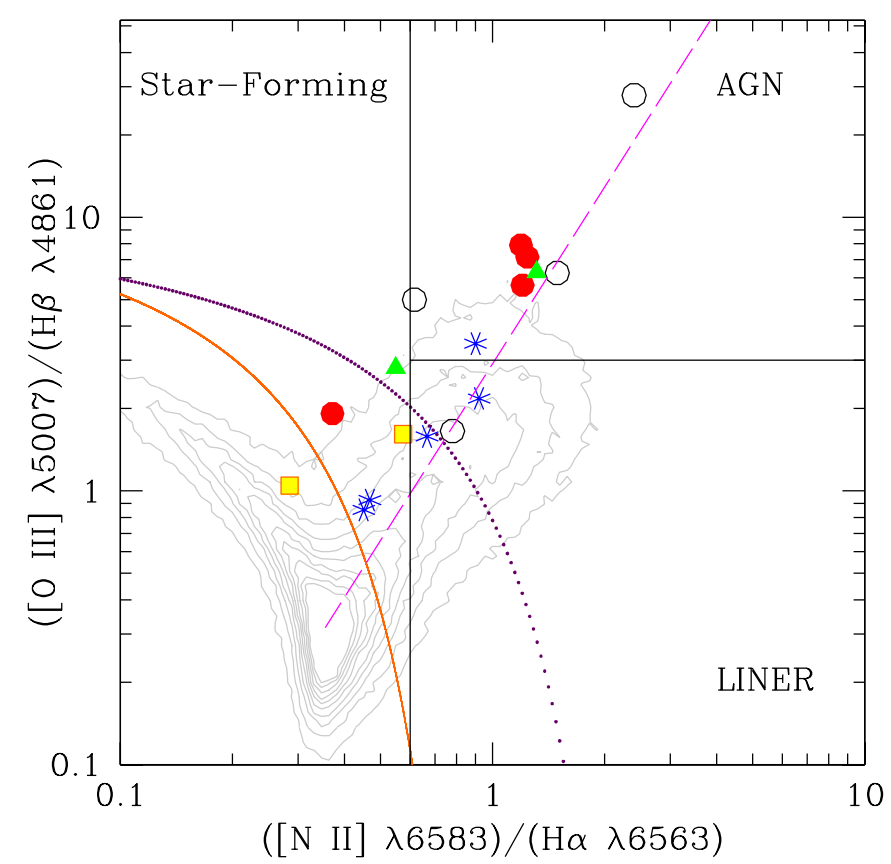

Figure 4. Narrow line BPT diagnostic diagram. The point type indicates morphology: early-type (red circles), spiral (blue stars), "probable" spiral (green triangles), indeterminate morphology (yellow squares), and no morphological data (black open circles). The contours mark the distribution of SDSS DR7 galaxies (after Kauffmann et al. 2003). With black solid lines, we make the traditional demarcations of (1) star formation if $[\mathrm{N}$ II $] / \mathrm{H} \alpha<0.6$, (2) AGNs for $[\mathrm{N} I I] / \mathrm{H} \alpha<0.6$ and $[\mathrm{O} \mathrm{III}] / \mathrm{H} \beta>3$, and (3) LINER (low-ionization nuclear emission region) when $\left[\mathrm{N}_{\mathrm{II}}\right] / \mathrm{H} \alpha<0.6$ and $[\mathrm{O} \mathrm{III}] / \mathrm{H} \beta<3$. The purple dotted curve gives the theoretical upper limit to the narrow-line ratios for star-forming regions given by Kewley et al. (2001). The orange solid curve gives a more conservative empirical relation that Kauffmann et al. (2003) which marks the lower boundary between star-forming galaxies and star-forming plus AGN (composite-type) galaxies. Furthermore, the magenta dashed diagonal line beginning at the locus of galaxies $([\mathrm{N} \mathrm{II}] / \mathrm{H} \alpha=-0.45$ and $[\mathrm{O} \mathrm{III}] / \mathrm{H} \beta=-0.5$ ) and extending toward the positive $[\mathrm{O} \mathrm{III}] / \mathrm{H} \beta$ axis and clockwise at an angle of $\phi=25^{\circ}$ marks a mixing line. Starting from the locus and increasing in distance away along the mixing line, AGNs become increasingly dominant. All PSQs show AGN activity in their spectra; however, PSQs fall along nearly the full extent of the mixing line indicating a broad range in relative contributions from star formation.

(A color version of this figure is available in the online journal.)

shows the traditional Baldwin et al. (1981, hereafter BPT) diagram, $[\mathrm{O}$ III $] / \mathrm{H} \beta$ versus $[\mathrm{N}$ II $] / \mathrm{H} \alpha$ flux ratios. Diagrams such as this one help us understand the nature of relative contributions of ionizing radiation from different sources. PSQs fall along and above nearly the full extent of the mixing line, which indicates the presence of an AGN but a wide range in the relative contribution of star formation.

\subsubsection{Starburst Properties}

The strongest correlation among starburst properties is between the age and mass of the post-starburst population (Figure 5). Just as in the case of AGN properties, Section 4.1.1, this correlation likely arises from a combination of demographics and selection effects. There is a lack of objects at large mass and young age. Such objects must exist and are likely dust enshrouded, seen in the infrared as luminous infrared galaxies (LIRGs; Sanders \& Mirabel 1996) or may be found as poststarburst galaxies if there is a significant time delay before the onset of AGN activity (see, e.g., Wild et al. 2010; Schawinski et al. 2009). The envelope in the upper left is the result of a selection effect: our luminosity limit.

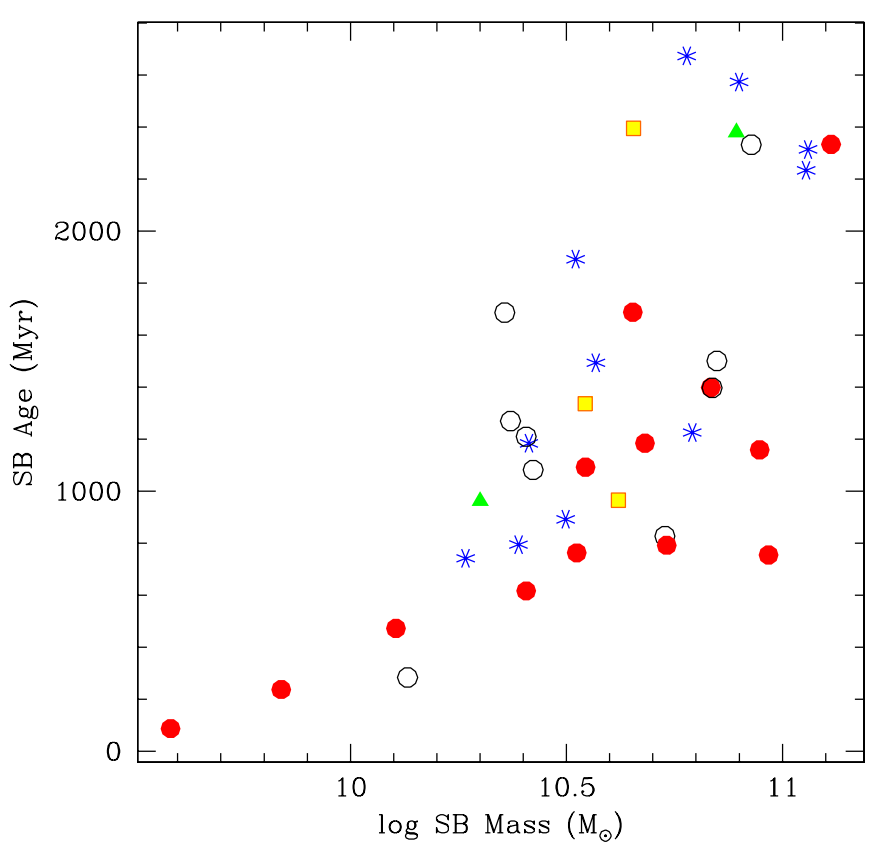

Figure 5. Starburst mass vs. age. The point type indicates morphology: early-type (red circles), spiral (blue stars), "probable" spiral (green triangles), indeterminate morphology (yellow squares), and no morphological data (black open circles). The dearth of points at low mass and old ages is due to the luminosity cut selection effect. However, the missing objects with young, massive starburst is unexpected. Thus, another class of objects such ULIRGs or post-starburst galaxies may be the parent population.

(A color version of this figure is available in the online journal.)

\subsubsection{AGN versus Starburst Properties}

We find that higher spectral indices (bluer AGNs) correlate with larger starburst masses. This could be a result of a degeneracy between the spectral index and scaling of the starburst. As the spectral index increases it has the effect of taking away light, in which case the scaling of the starburst must make up the difference. Furthermore, if we assume a spectral index more typical of AGNs, we might expect a stronger correlation (e.g., Francis 1993 find $\alpha \sim 1.5$ with 0.5 scatter versus our 1.0). However, while the starburst template continuum guides our fitting, the Balmer region (i.e., Balmer break and absorption lines) of the spectra governs the quality of the starburst fit (see Figure 1).

Perhaps the most interesting result from this analysis is the lack of significant correlations between parameters which might have been suggested by merger-induced evolutionary scenarios (e.g., Di Matteo et al. 2005; Springel et al. 2005; Hopkins et al. 2006). Specifically, AGN luminosity (and/or Eddington ratio) should decline following a merger-triggered fueling event as the age of the starburst population increases. If there is a single mechanism driving both AGNs and starburst activity, naturally leading to correlations between their properties, then our results present a problem for such models. Our selection effects that limit parameter space could limit our ability to see correlations or there could be a delay in AGN triggering or multiple types of triggering events. For the latter reason, we also perform analysis by morphological classification.

\subsection{Dependence on Morphological Class}

We investigate the relationships among the morphology and fundamental (fitted and derived) properties of the AGN, narrow-line emission, and starburst. Visual classifications were 
Table 8

Population Tests

\begin{tabular}{|c|c|c|c|c|c|c|c|c|c|c|c|}
\hline \multirow[t]{2}{*}{ Property } & \multicolumn{3}{|c|}{ Total } & \multicolumn{3}{|c|}{ Early-type } & \multicolumn{3}{|c|}{ Spirals } & \multirow[b]{2}{*}{$W^{\mathrm{d}}$} & \multirow[b]{2}{*}{$P(\%)$} \\
\hline & $\mu^{\mathrm{a}}$ & $\sigma^{\mathrm{b}}$ & Number $^{\mathrm{c}}$ & $\mu$ & $\sigma$ & Number & $\mu$ & $\sigma$ & Number & & \\
\hline$z$ & 0.32 & 0.05 & 38 & 0.33 & 0.05 & 13 & 0.31 & 0.05 & 13 & $\ldots \ldots$ & $\ldots \ldots$ \\
\hline $\log \lambda L_{\lambda}(5100 \AA)$ & 43.94 & 0.26 & 38 & 44.11 & 0.23 & 13 & 43.77 & 0.24 & 13 & -3.051 & 0.114 \\
\hline$\alpha$ & 1.00 & 0.66 & 38 & 1.11 & 0.66 & 13 & 1.06 & 0.70 & 13 & $\ldots \ldots$ & $\ldots \ldots$ \\
\hline$f_{\text {nuc }}$ & 0.55 & 0.13 & 38 & 0.57 & 0.15 & 13 & 0.51 & 0.12 & 13 & $\ldots \ldots$ & $\ldots \ldots$ \\
\hline $\log L_{\mathrm{Tot}}$ & 43.93 & 0.20 & 38 & 44.08 & 0.18 & 13 & 43.81 & 0.16 & 13 & -3.359 & 0.039 \\
\hline $\log L_{\mathrm{AGN}}$ & 43.65 & 0.24 & 38 & 43.82 & 0.23 & 13 & 43.51 & 0.22 & 13 & -3.205 & 0.068 \\
\hline $\log L_{S B}$ & 43.57 & 0.20 & 38 & 43.69 & 0.20 & 13 & 43.50 & 0.14 & 13 & -2.590 & 0.480 \\
\hline $\log M_{\mathrm{BH}} \mathrm{Mg}_{\mathrm{II}}$ & 7.99 & 0.37 & 29 & 8.04 & 0.40 & 12 & 8.08 & 0.39 & 9 & $\ldots \ldots$ & \\
\hline$L / L_{\text {Edd }} \mathrm{Mg}$ II & 0.07 & 0.06 & 29 & 0.08 & 0.06 & 12 & 0.05 & 0.05 & 9 & $\ldots \ldots$ & $\ldots$ \\
\hline $\log M_{\mathrm{BH}} \mathrm{H} \beta$ & 8.27 & 0.41 & 30 & 8.28 & 0.39 & 13 & 8.49 & 0.45 & 7 & $\ldots \ldots$ & \\
\hline$L / L_{\text {Edd }} \mathrm{H} \beta$ & 0.04 & 0.04 & 30 & 0.05 & 0.04 & 13 & 0.02 & 0.02 & 7 & $\ldots \ldots$ & \\
\hline $\log M_{\mathrm{BH}} \mathrm{H} \alpha$ & 8.25 & 0.38 & 21 & 8.18 & 0.29 & 6 & 8.30 & 0.38 & 9 & $\ldots \ldots$ & $\ldots$ \\
\hline$L / L_{\text {Edd }} \mathrm{H} \alpha$ & 0.03 & 0.02 & 21 & 0.04 & 0.02 & 6 & 0.02 & 0.02 & 9 & $\ldots \ldots$ & \\
\hline $\log M_{\mathrm{BH}} \mathrm{H} \alpha-\mathrm{H} \beta$ & 8.28 & 0.38 & 21 & 8.21 & 0.29 & 6 & 8.34 & 0.39 & 9 & $\ldots \ldots$ & \\
\hline$L / L_{\text {Edd }} \mathrm{H} \alpha-\mathrm{H} \beta$ & 0.03 & 0.02 & 21 & 0.04 & 0.02 & 6 & 0.02 & 0.02 & 9 & $\ldots \ldots$ & $\ldots \ldots$ \\
\hline $\log M_{\mathrm{BH}}$ Adopted & 8.17 & 0.36 & 37 & 8.19 & 0.33 & 13 & 8.20 & 0.38 & 13 & $\ldots \ldots$ & $\ldots$ \\
\hline$L / L_{\text {Edd }}$ Adopted & 0.05 & 0.05 & 37 & 0.06 & 0.04 & 13 & 0.03 & 0.04 & 13 & $\ldots \ldots$ & \\
\hline $\log$ SB Mass & 10.58 & 0.33 & 38 & 10.53 & 0.45 & 13 & 10.65 & 0.28 & 13 & $\ldots \ldots$ & $\ldots \ldots$ \\
\hline SB Age & 1321.75 & 689.74 & 38 & 967.60 & 609.97 & 13 & 1643.17 & 724.48 & 13 & 2.333 & 0.982 \\
\hline$[\mathrm{O} \mathrm{III}] / \mathrm{H} \beta$ & 4.83 & 5.29 & 32 & 6.56 & 4.05 & 10 & 2.54 & 1.59 & 11 & -3.053 & 0.113 \\
\hline$[\mathrm{N}$ II $] / \mathrm{H} \alpha$ & 1.06 & 0.92 & 21 & 1.57 & 1.47 & 6 & 0.73 & 0.27 & 9 & $\ldots \ldots$ & $\ldots \ldots$ \\
\hline$[\mathrm{O} \mathrm{III}] /[\mathrm{O} \mathrm{II}]$ & 2.31 & 1.60 & 33 & 2.79 & 1.60 & 11 & 1.59 & 0.77 & 11 & $\ldots \ldots$ & $\ldots$ \\
\hline$[\mathrm{Ne} \mathrm{v}] /[\mathrm{Ne} \mathrm{III}]$ & 1.35 & 2.04 & 33 & 2.07 & 2.13 & 11 & 1.61 & 0.96 & 11 & $\ldots \ldots$ & \\
\hline Fe II/[O III $]$ & 0.01 & 0.02 & 12 & 0.01 & 0.02 & 6 & 0.03 & 0.03 & 2 & $\ldots \ldots$ & $\ldots \ldots$ \\
\hline
\end{tabular}

Notes.

a The mean of the population.

$\mathrm{b}$ The standard deviation of the population.

$\mathrm{c}$ The number of objects that were used to calculate mean, standard deviation, and/or population statistics.

${ }^{\mathrm{d}}$ When statistical differences in sample means exist at below the $1 \%$ level we include the nonparametric Mann-Whitney statistic and its associated $P$-Value.

performed by three of the authors and the results are presented in Cales et al. (2011). Generally, arms and bars distinguish spiral galaxies while smooth, somewhat featureless (i.e., lacking arms/bars), light distributions identify early-type galaxies. We note that the early-type hosts are allowed to show tidal features although sometimes disturbances make classification difficult, resulting in an indeterminate classification. In Table 8, we list the mean, standard deviation, and number of objects for our basic properties for the total sample, and early-type and spiral (including "probable" spirals) subpopulations. When reliable statistical differences in sample means exist at $P$ the Values below the $1 \%$ level (better than $2.58 \sigma$ ) we include the nonparametric Mann-Whitney statistic and its associated $P$-Value and give their distributions in Figure 6. For reference, the mean, standard deviation, and number of objects for the total sample are also given.

Due to the small number of objects in the subpopulations and the lack of significant differences below the $1 \%$ level between the disturbed and undisturbed populations, we do not present population statistics for classifications of this type.

The significant differences for fundamental parameters within the morphology subclasses are as follows.

1. AGNs. The PSQs hosted in early-type galaxies have statistically significant higher total luminosities than spiral-hosted PSQs, and this appears to be somewhat more driven by differences in AGN luminosity although starburst luminosities also differ. This is supported by various methods of measuring the PSQ luminosity and its components (i.e., $L_{\mathrm{Tot}}$, $L_{\mathrm{AGN}}$, and $L_{\mathrm{SB}}$; see Figures 6(a)-(c)). We note that AGN luminosity increases with $\mathrm{BH}$ mass and/or accretion rate. While there are no statistically significant differences between the subpopulations for Eddington fraction, $\mathrm{BH}$ mass, or starburst mass, it is possible that the AGN with earlytype hosts tend to have higher luminosities because they have higher Eddington ratios (Figures 6(d)-(f)).

2. NLR. Figure 6(e) shows that the PSQs hosted in early-type galaxies have larger $[\mathrm{O} \mathrm{III}] / \mathrm{H} \beta$ than spiral-hosted PSQs, and we interpret this as differences in the hardness of the ionizing continuum reflecting the relative contributions of the AGN and star formation. The spiral PSQs have more ongoing star formation.

3. Starburst. The PSQs hosted in early-type galaxies tend to have younger starburst ages from our fitting results than the PSQs hosted by spirals (Figure 6(f)). This may be interpreted in different ways. Some possible explanations of this are: (1) the starburst luminosities are smaller in the spiral-hosted PSQs and our single age fits may be skewed by the presence of an older stellar population, (2) chance snapshot of unassociated starburst and AGN activity in spiral hosts with large starburst ages, or (3) longer timescales between triggering of the AGN and starburst in spiral PSQs. There may be no clear interpretation of this effect without additional data.

\subsection{Spectral Diagnostics for Mode of Growth}

PSQs appear to display both merger-driven and secular forms of galaxy evolution. They are a heterogeneous population hosted by both early-type and spiral galaxies, some of which 


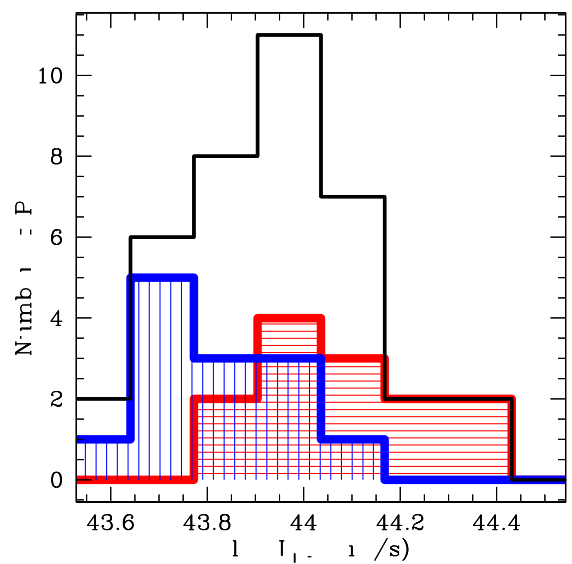

(a)

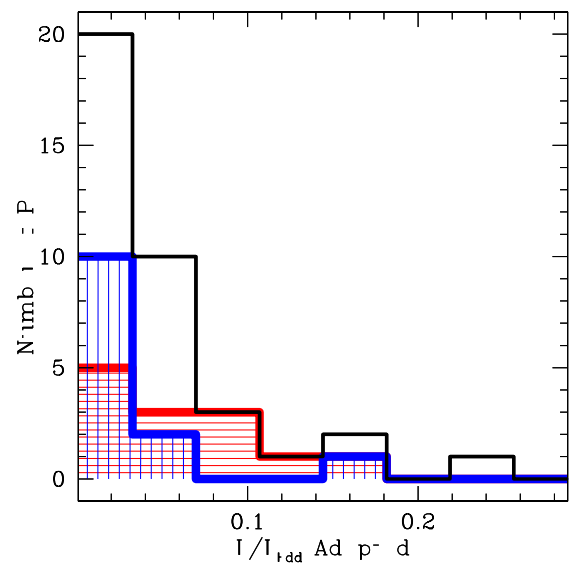

(d)

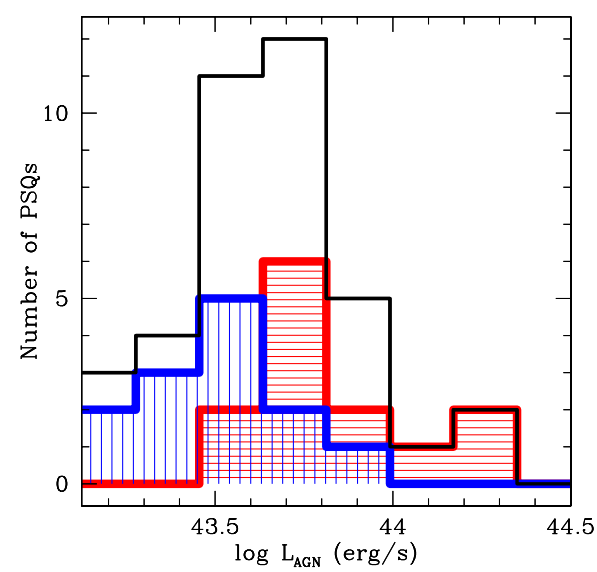

(b)

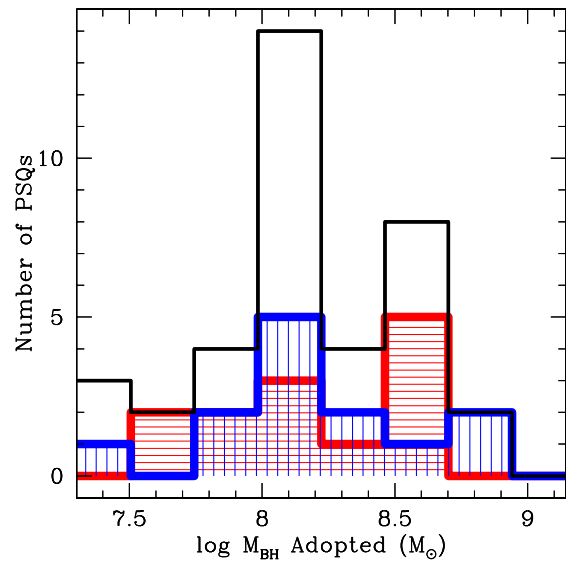

(e)

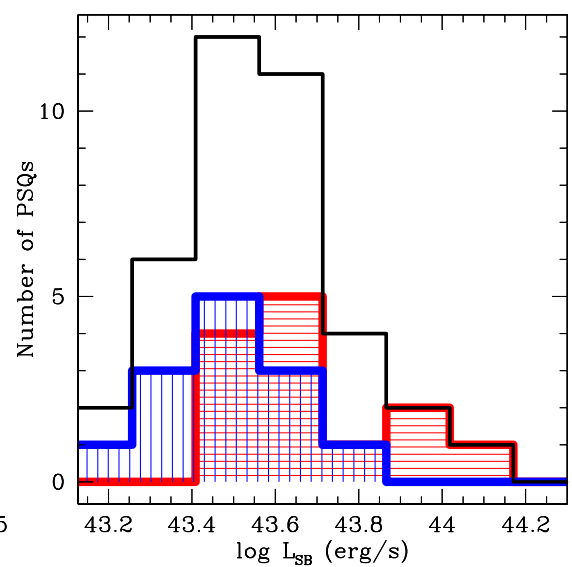

(c)

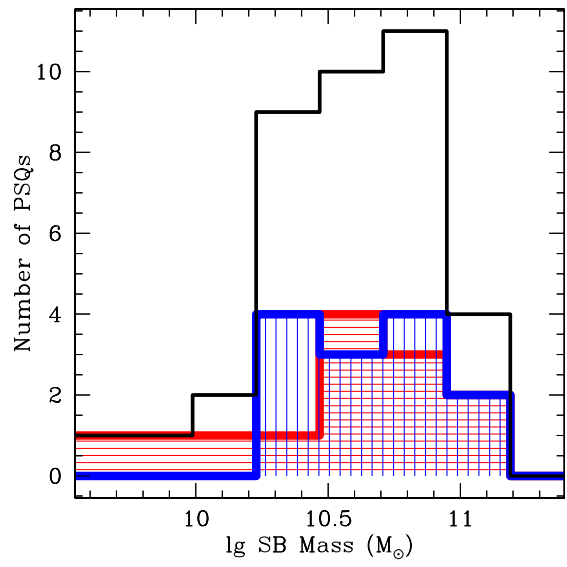

(f)

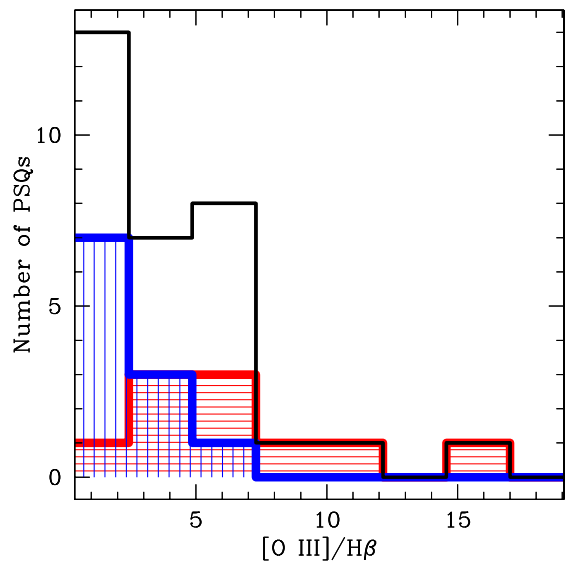

(g)

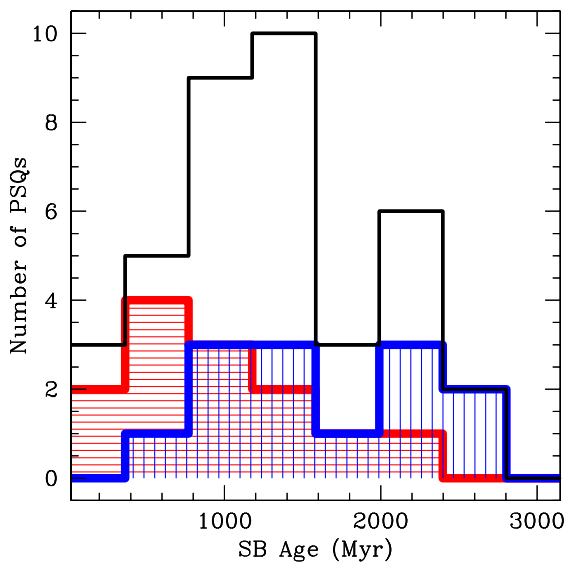

(h)

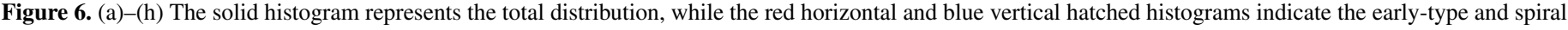

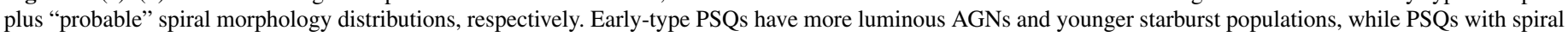
hosts are consistent with ongoing star formation.

(A color version of this figure is available in the online journal.)

appear to be results of major mergers while others exist in isolated systems. C11 postulates that the early-type PSQs may be the low $-z$ analogs of luminous merger-induced $z \sim$ 2 quasars. Furthermore, at least some of the spiral PSQs are likely undergoing secular evolution. This conclusion is consistent with current theoretical frameworks which argue for two fundamentally different fueling mechanisms (i.e., merger induced versus secular) responsible for mutual SMBH-bulge growth at the characteristic dividing line of quasar-Seyfert luminosities (Hopkins \& Hernquist 2009).

We explored issues of the relative contributions of AGNs and star formation to powering narrow-emission lines previously in the context of the BPT diagram. We have also found differences between a number of properties and morphological subtype. We 


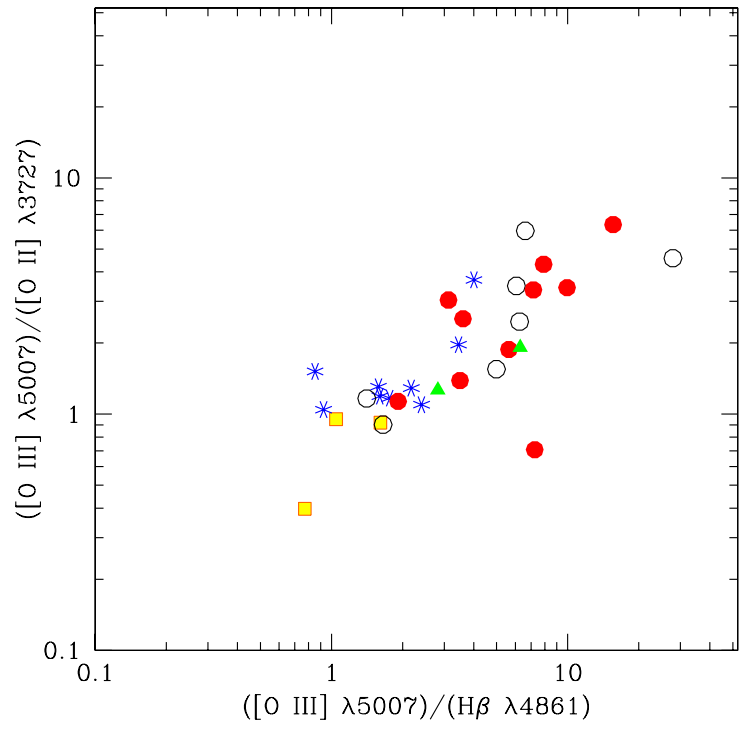

(a)

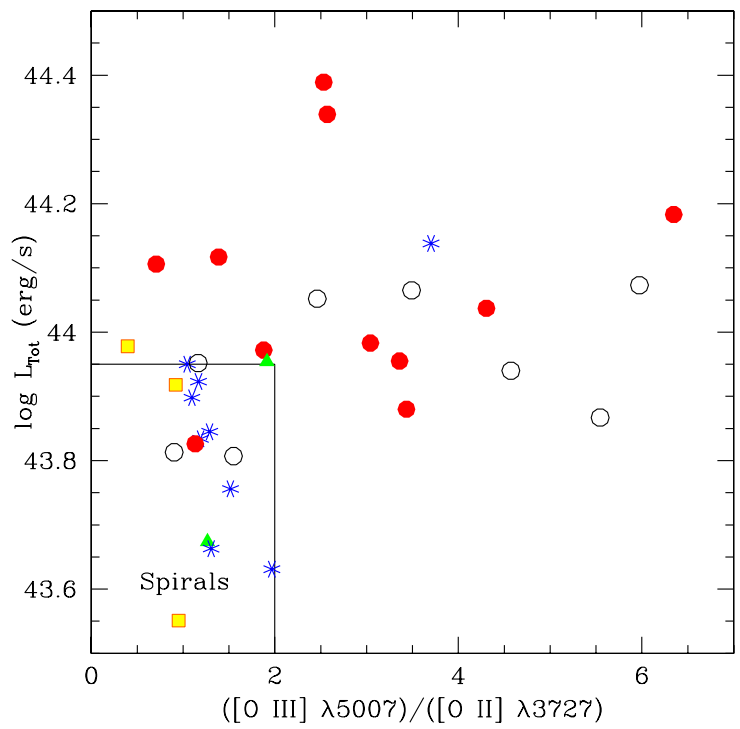

(c)

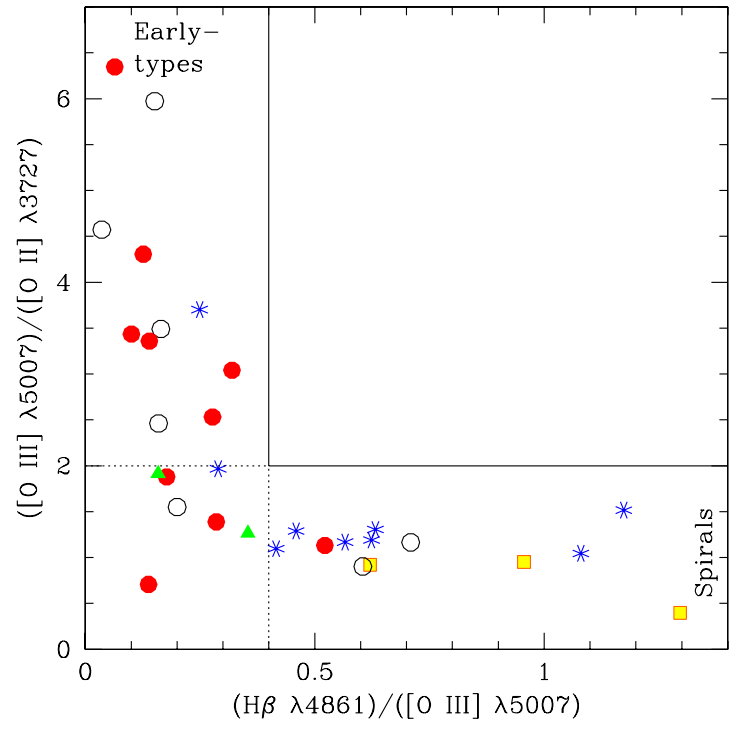

(b)

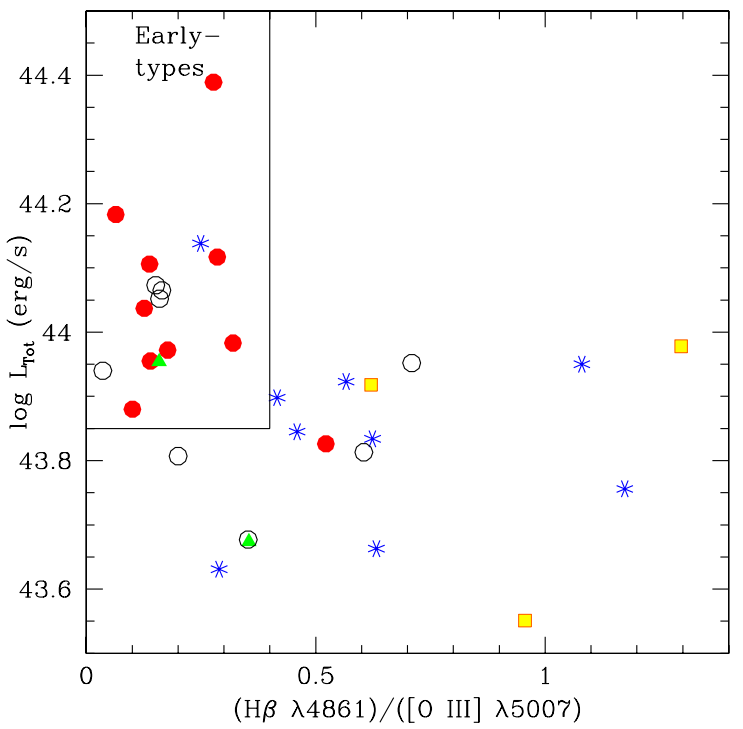

(d)

Figure 7. Diagnostic diagrams featuring [O III]/[O II], [O III]/H $\beta$, and $L_{\text {Tot }}$. The point type indicates morphology: early-type (red circles), spiral (blue stars), "probable" spiral (green triangles), indeterminate morphology (yellow squares), and no morphological data (black open circles). (a) The $\log -\log [\mathrm{O}$ III]/[O II] vs. [O III]/H $\beta$ plot. (b) Linear $\left[\mathrm{O}\right.$ III] $/\left[\mathrm{O}\right.$ II] vs. $\mathrm{H} \beta /\left[\mathrm{O}\right.$ III]. (c) $L_{\text {Tot }}$ vs. [O III] $/\left[\mathrm{O}\right.$ II]. (d) $L_{\mathrm{Tot}} \mathrm{vs}$. $\mathrm{H} \beta /[\mathrm{O}$ III]. PSQs with early-type and spiral hosts lie in very different regions of the three-dimensional space given by $L_{\mathrm{Tot}},[\mathrm{O} \mathrm{III}] /[\mathrm{O} \mathrm{II}]$ and $\mathrm{H} \beta /[\mathrm{O}$ III $]$. We note where early-type and spiral PSQs lie according to our selection criteria.

(A color version of this figure is available in the online journal.)

can combine this information to create observational selection criteria to select PSQs as a function of galaxy host type without requiring high spatial resolution imaging. With the exception of a rare outlier, we can use a combination of $\mathrm{H} \beta /[\mathrm{O} \mathrm{III}]$, [O III]/ [O II], and $L_{\text {Tot }}$ to distinguish host galaxy type. Figure 7 shows several plots involving these quantities and where the objects of different classification fall. Using these we establish the following parameters to select PSQs by morphological subtype:

1. Early-type: $L_{\mathrm{Tot}}>10^{43.85} \mathrm{erg} \mathrm{s}^{-1}+\mathrm{H} \beta /\left[\mathrm{O}_{\mathrm{III}}\right]<0.4$;

2. Spiral: $L_{\mathrm{Tot}}<10^{43.95} \mathrm{erg} \mathrm{s}^{-1}+\left[\mathrm{O}_{\mathrm{III}}\right] /\left[\mathrm{O}_{\mathrm{II}}\right]<2.0$.

While it has been suggested that major-mergers are not important drivers of AGN activity in the local downsized universe (Cisternas et al. 2011), such objects do exist primarily among quasars and may be valuable to study as analogs of luminous high-redshift quasars (Canalizo et al. 2007; Bennert et al. 2008, 2010). Studies of local AGNs $(z \sim 0.05)$ have also found two modes of growth distinguished by morphology. Schawinski et al. (2010b) find that the least massive SMBH population of early-type hosts is growing while the most massive SMBH population of late-type hosts is growing. Furthermore, Schawinski et al. (2010a) trace a correlation between the merger fraction of early-type hosts and an evolutionary sequence involving starbursts and AGNs, such that, even in the local universe, early-type galaxies are still merger induced. This has important ramifications for future studies involving galaxy evolution and should guide the path of such studies.

\section{SUMMARY}

We performed AGN-host spectral decomposition of 38 PSQs, 29 of which have morphological classifications from $\mathrm{C} 11$. We characterized the host starburst masses and ages, and the AGN $\mathrm{BH}$ masses and Eddington fractions, with the aim of improving 


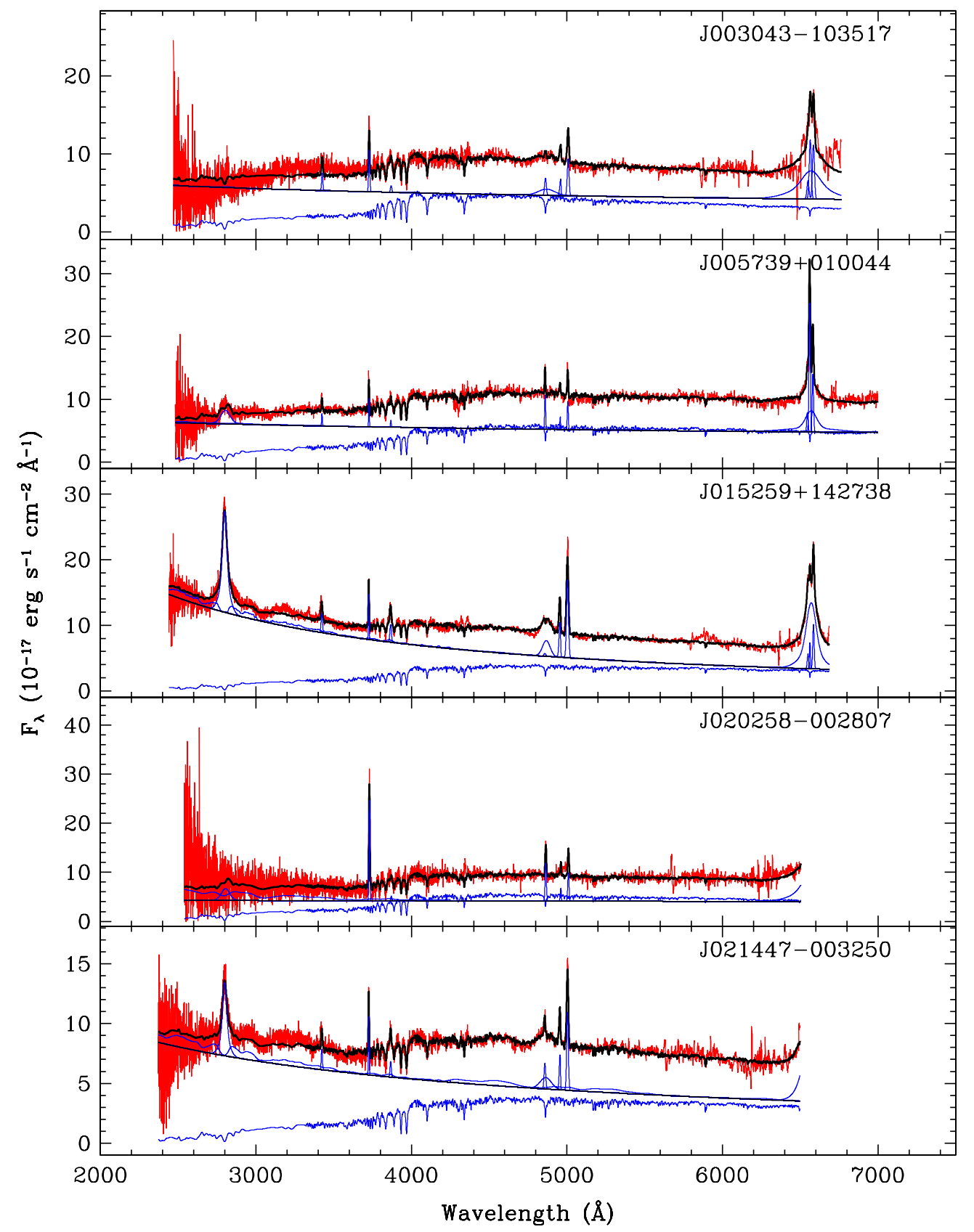

Figure 8. Spectra of Fitting Results.

(A color version of this figure is available in the online journal.)

our understanding of the mutual evolution of luminous AGNs and their hosts.

1. Black hole properties. The PSQs have $M_{\mathrm{BH}} \sim 10^{7.5}-10^{8.5}$ $M_{\odot}$ which are accreting at $\sim 1 \%-10 \%$ of Eddington luminosity.

2. Starburst properties. The PSQs have massive starbursts $\sim 10^{10}-10^{11} M_{\odot}$ that span a range of ages $\sim 200-2000 \mathrm{Myr}$.

3. We find no strong correlations linking $M_{\mathrm{BH}}$ and starburst properties.

4. The PSQ sample lacks young, massive starbursts that are likely obscured LIRGs that we cannot select optically or are seen as post-starburst galaxies.

5. Narrow-line emission. When plotted on traditional BPT diagrams, PSQs fall along and slightly above the mixing line showing a wide range in the relative contributions of AGNs and star formation.
6. Morphology. Early-type PSQs have significantly stronger AGN luminosities, younger ISB ages, and narrow-line ratios indicative of harder photoionizing continua when compared to spiral PSQs.

7. Morphological selection of PSQs. We determine that the selection criterion for (1) early-type PSQs of $L_{\mathrm{Tot}}>$ $10^{43.85} \mathrm{erg} \mathrm{s}^{-1}$ and $\mathrm{H} \beta /[\mathrm{O} \mathrm{III}]<0.4$ and (2) spiral PSQs of $L_{\text {Tot }}<10^{43.95} \mathrm{erg} \mathrm{s}^{-1}$ and [O III] $/[\mathrm{O}$ II $]<2.0$ is efficient for determining morphology of the PSQ sample.

8. We conclude that the PSQ sample displays two distinct mechanisms for joint AGNs and starburst activity. The higher luminosity early-type PSQs appear to be the product of major mergers, show little current star formation, and may be identified as the low- $z$ analogs of the luminous and likely merger-induced high-redshift quasars. PSQs hosted in spirals likely represent a lower luminosity mode 


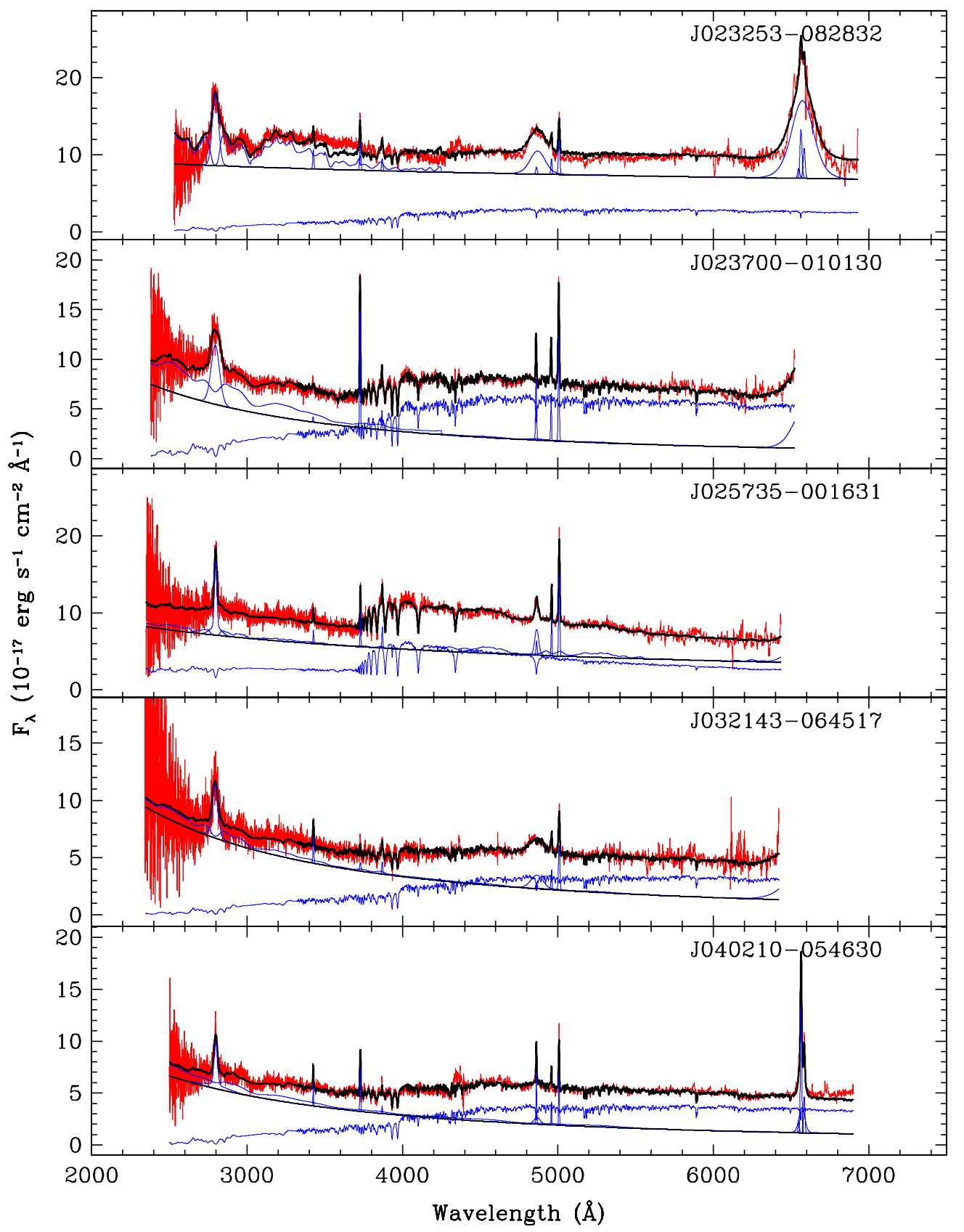

Figure 8. (Continued)

of activity, such as "Seyfert mode" or secular activity, triggered by internal processes, e.g., bars, or external triggers, e.g., harassment.

In order to further explore possible correlations between AGNs and starburst properties, we intend to study a larger sample of PSQs with a broader range in AGNs and starburst strengths. For example, plotting PSQs on the $M_{\mathrm{BH}}-\sigma_{*}$ relationship may be helpful in better understanding their role in massive galaxy evolution (Hiner et al., 2012). Another way to do this is to explore lower luminosity, lower redshift objects of the Brotherton et al. (2007) catalog in conjunction with morphologies from Galaxy Zoo and spectral fitting of only the highest quality SDSS spectra.

We acknowledge support from NASA through the LTSA grant NNG05GE84G. Z.S. acknowledges support from the National Natural Science Foundation of China through grant 10633040 and support by Chinese 973 Program 2007CB815405. G.C. acknowledges support from the National Science Foundation, under grant number AST 0507450. S. L. Cales was supported in part by NASA Headquarters under the NASA Earth and Space Science Fellowship Program (Grant NNX08AX07H), in part by the National Science Foundation GK-12 Program (Project 0841298), and also in part by ALMA-CONICYT program 31110020. A.D. acknowledges support from the Southern California Center for Galaxy Evolution, a multi-campus research program funded by the University of California Office of Research.

\section{APPENDIX}

\section{SPECTRA OF FITTING RESULTS}

Spectral decomposition of AGNs and post-starburst stellar population is shown in Figure 8. The red line is the data. The 


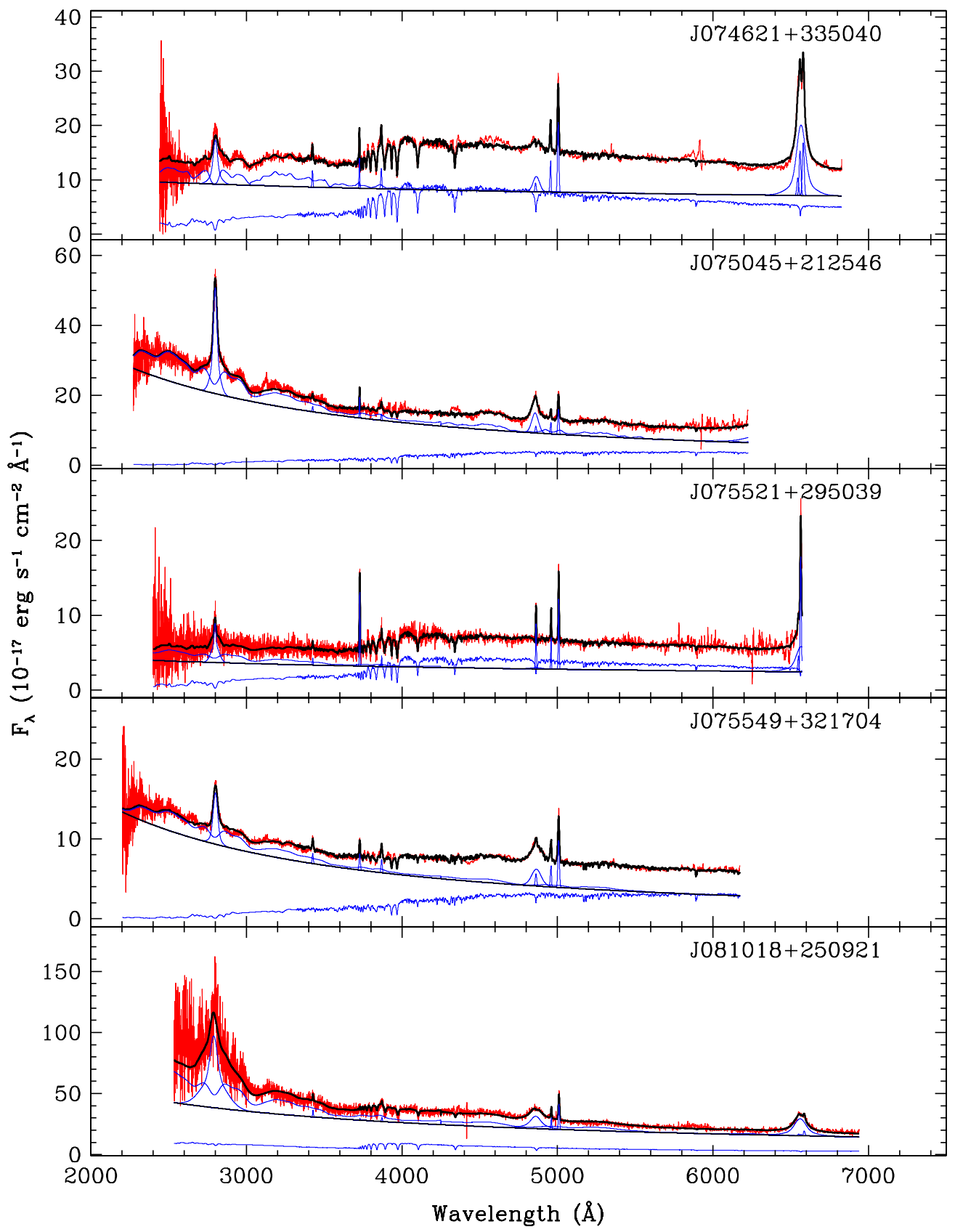

Figure 8. (Continued) 


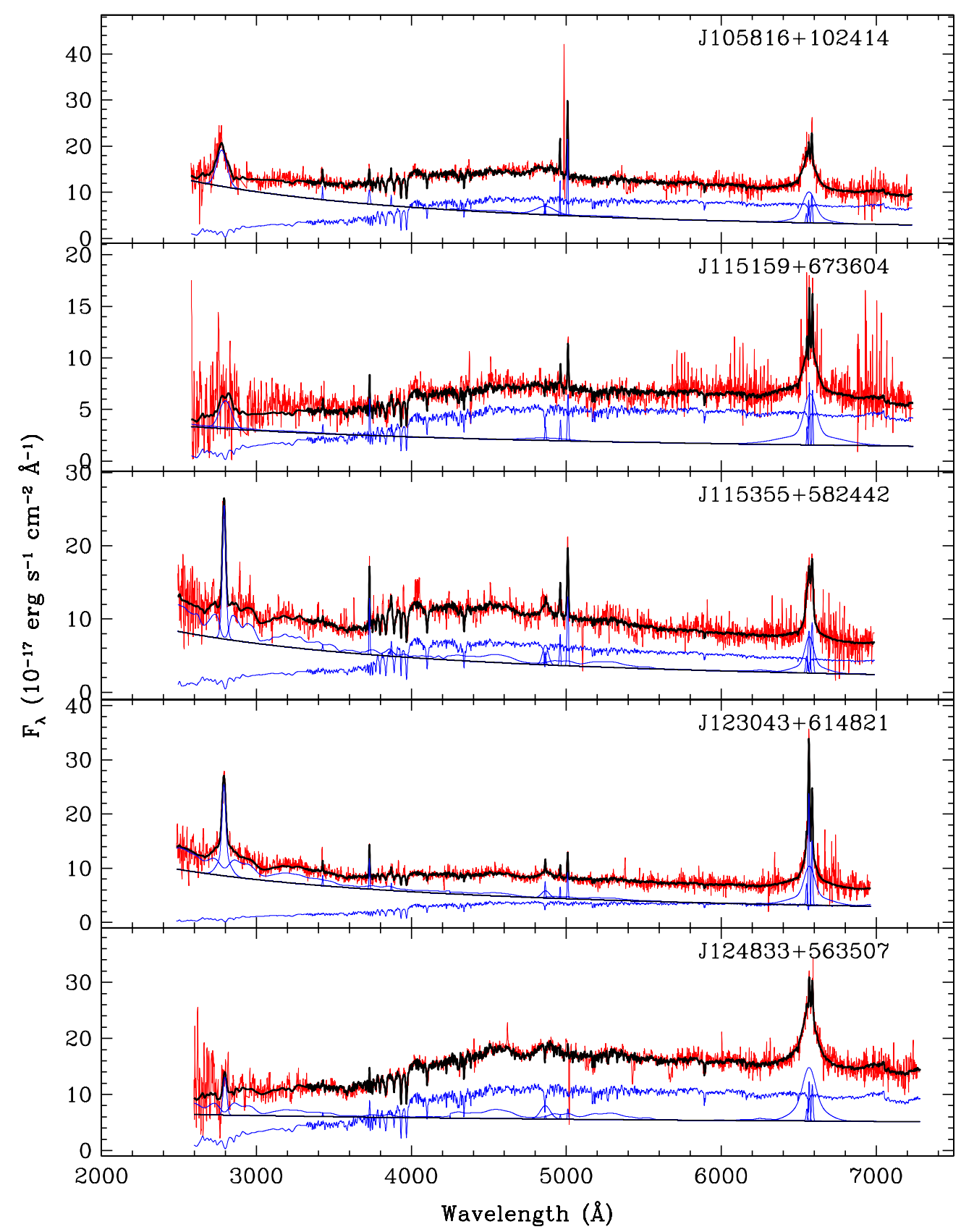

Figure 8. (Continued) 


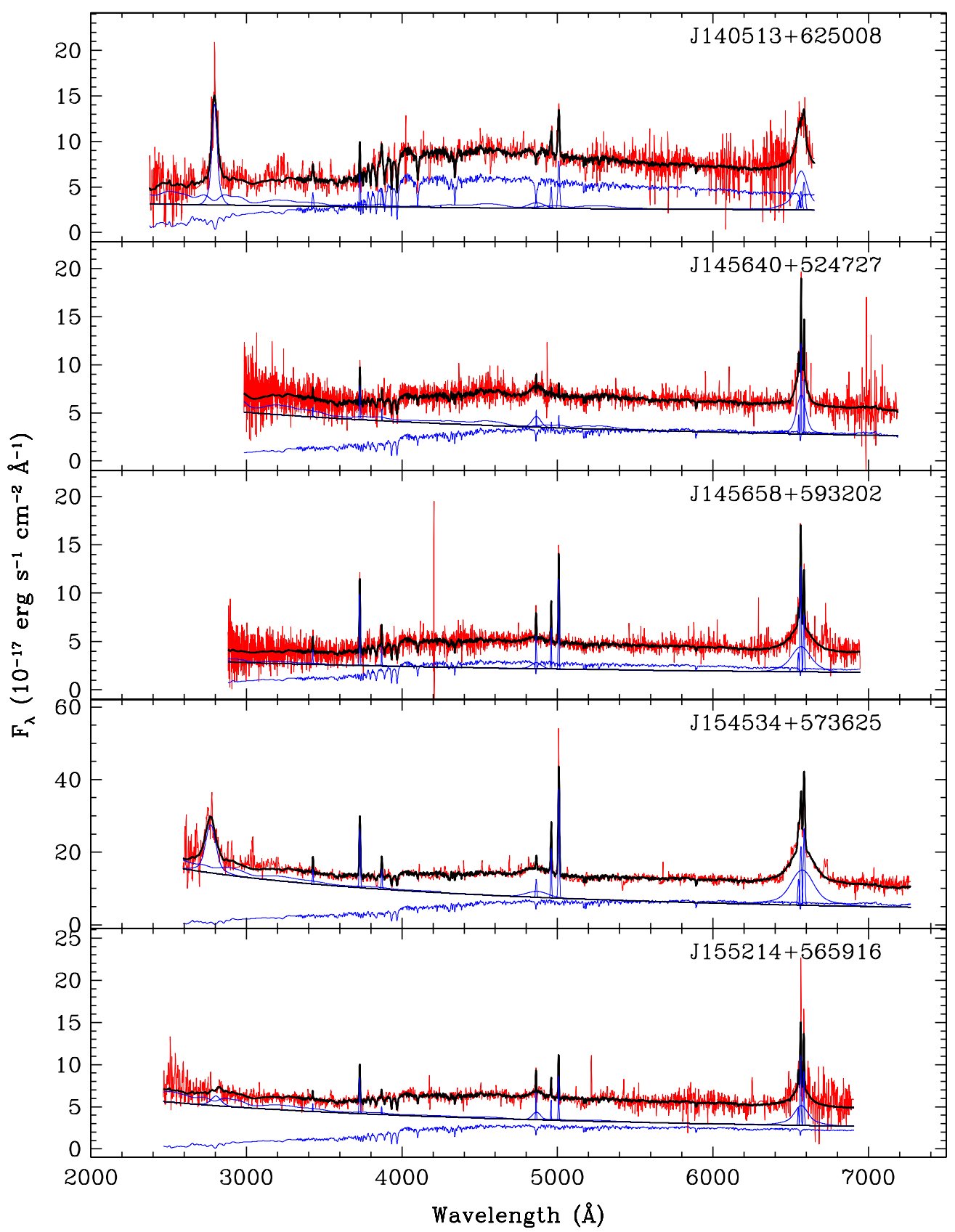

Figure 8. (Continued) 


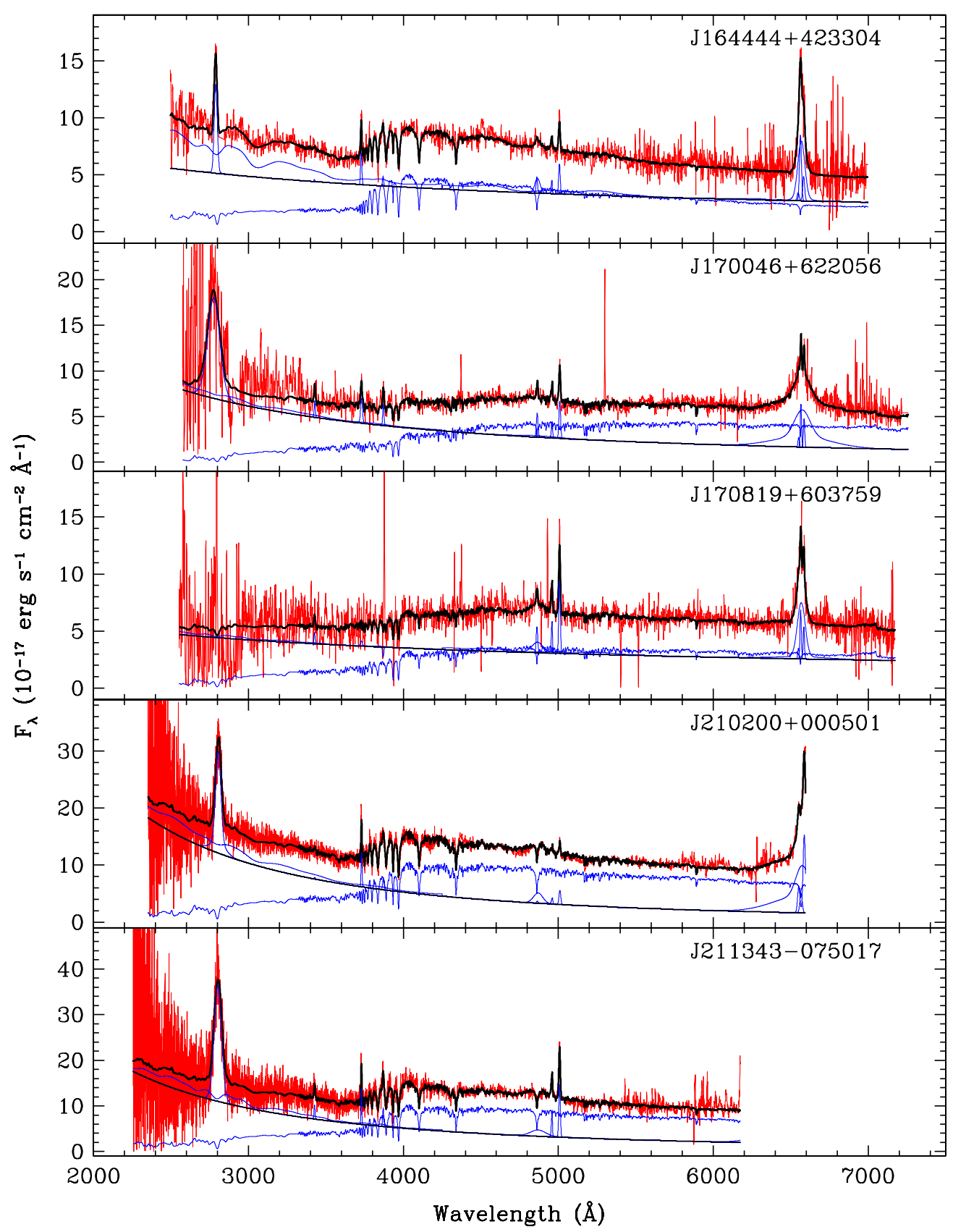

Figure 8. (Continued) 


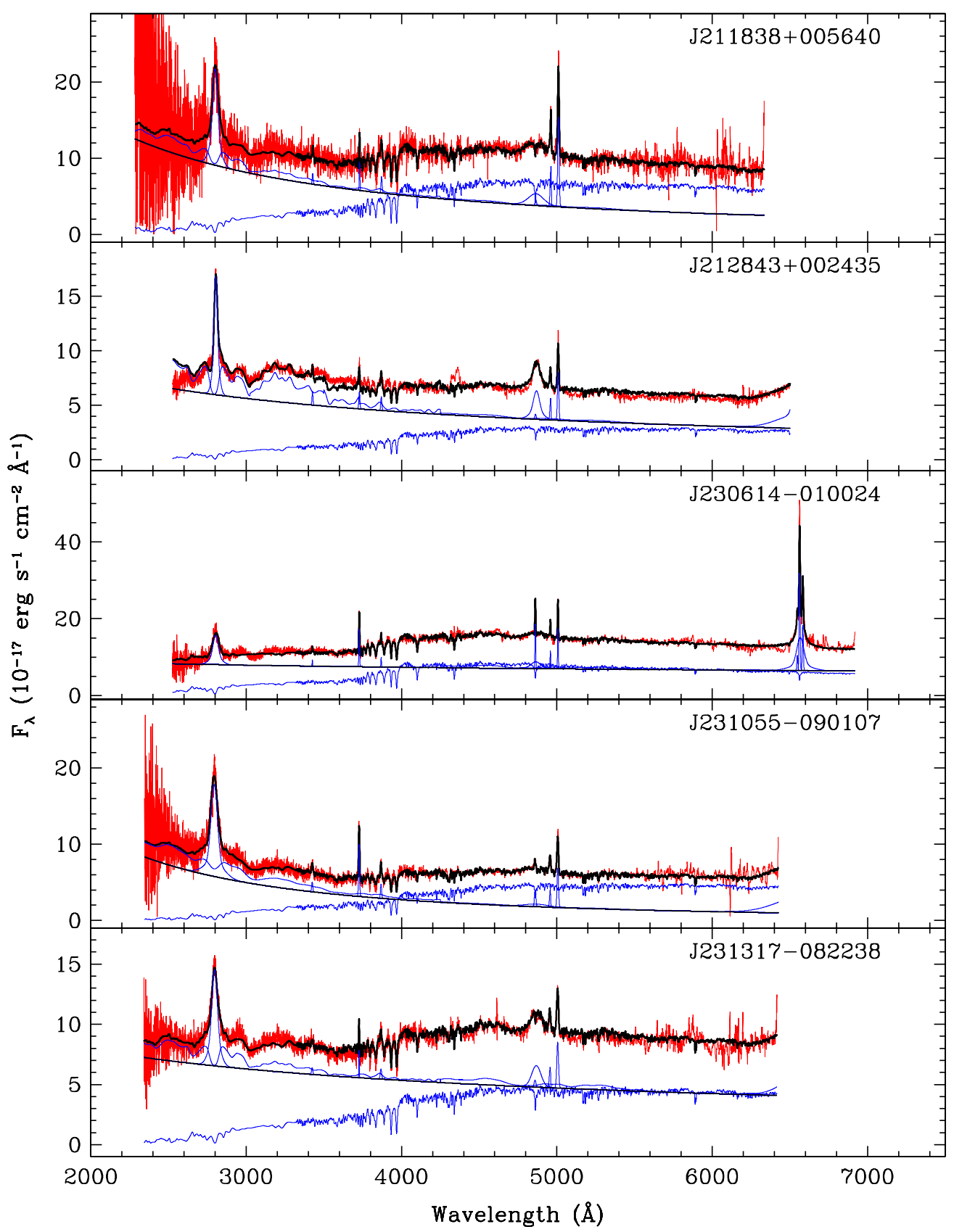

Figure 8. (Continued) 


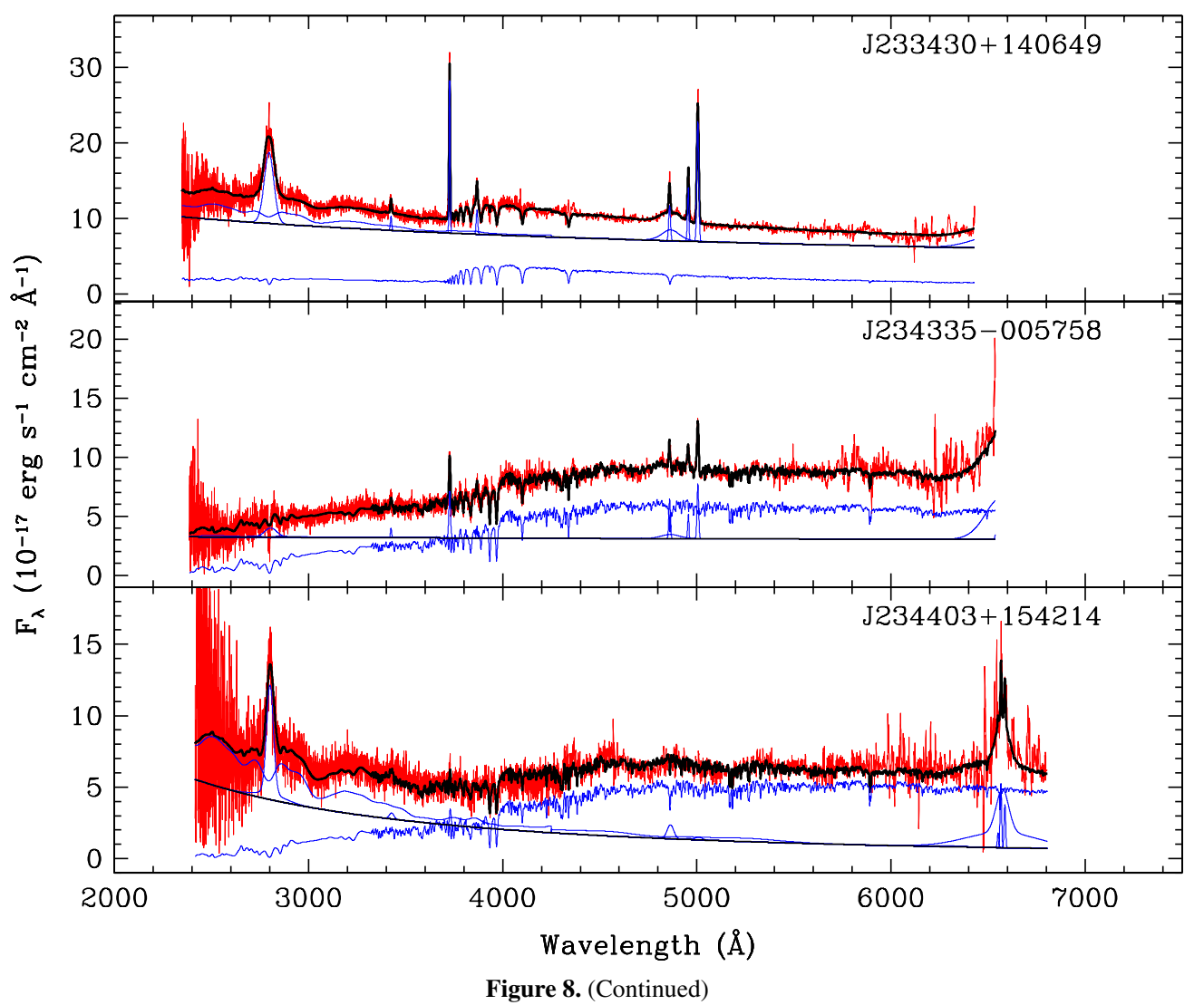

blue lines make up the components used in the fitting. For ease of interpretation we plot the emission lines and Fe II templates above the power law. The black line is the model fit to the data.

\section{REFERENCES}

Abazajian, K., Adelman-McCarthy, J. K., Ageros, M. A., et al. 2005, AJ, 129,1755

Baldwin, J. A., Phillips, M. M., \& Terlevich, R. 1981, PASP, 93, 5

Bennert, N., Canalizo, G., Jungwiert, B., et al. 2008, ApJ, 677, 846

Bennert, V. N., Auger, M. W., Treu, T., Woo, J.-H., \& Malkan, M. A. 2011, ApJ, 742, 107

Bennert, V. N., Treu, T., Woo, J., et al. 2010, ApJ, 708, 1507

Boroson, T. A., \& Green, R. F. 1992, ApJS, 80, 109

Brotherton, M. S., Grabelsky, M., Canalizo, G., et al. 2002, PASP, 114, 593

Brotherton, M. S., van Breugel, W., Stanford, S. A., et al. 1999, ApJL, 520, 87

Brotherton, M. S., et al. 2007, BAAS, 39, 95

Cales, S. L., Brotherton, M. S., Shang, Z., et al. 2011, ApJ, 741, 106

Calzetti, D., Kinney, A. L., \& Storchi-Bergmann, T. 1994, ApJ, 429, 582

Canalizo, G., Bennert, N., Jungwiert, B., et al. 2007, ApJ, 669, 801

Canalizo, G., Stockton, A., Brotherton, M. S., \& van Breugel, W. 2000, AJ, 119,59

Cardelli, J. A., Clayton, G. C., \& Mathis, J. S. 1989, ApJ, 345, 245

Chabrier, G. 2003, PASP, 115, 763

Cisternas, M., Jahnke, K., Inskip, K. J., et al. 2011, ApJ, 726, 57

Di Matteo, T., Springel, V., \& Hernquist, L. 2005, Natur, 433, 604

Dressler, A., \& Gunn, J. E. 1983, ApJ, 270, 7

Falkenberg, M. A., Kotulla, R., \& Fritze, U. 2009, MNRAS, 397, 1940

Ferrarese, L., \& Merritt, D. 2000, ApJL, 539, 9

Francis, P. J. 1993, ApJ, 407, 519

Gebhardt, K., Bender, R., Bower, G., et al. 2000a, ApJL, 539, 13

Gebhardt, K., Kormendy, J., Ho, L. C., et al. 2000b, ApJL, 543, 5

Georgakakis, A., Nandra, K., Yan, R., et al. 2008, MNRAS, 385, 2049

Goto, T. 2006, MNRAS, 369, 1765

Greene, J. E., Peng, C. Y., \& Ludwig, R. R. 2010, ApJ, 709, 937

Hasinger, G. 2008, A\&A, 490, 905

Heckman, T. M., Kauffmann, G., Brinchmann, J., et al. 2004, ApJ, 613, 109

Hiner, K. D., Canalizo, G., Wold, M., Brotherton, M. S., \& Cales, S. L. 2012, ApJ, 756, 162
Hopkins, P. F., \& Hernquist, L. 2009, ApJ, 694, 599

Hopkins, P. F., Hernquist, L., Cox, T. J., Robertson, B., \& Springel, V. 2006, ApJS, 163, 50

Kauffmann, G., Heckman, T. M., Tremonti, C., et al. 2003, MNRAS, 346, 1055 Kewley, L. J., Dopita, M. A., Sutherland, R. S., Heisler, C. A., \& Trevena, J. 2001, ApJ, 556, 121

Kocevski, D. D., Lemaux, B. C., Lubin, L. M., et al. 2011, ApJL, 737, 38

Kocevski, D. D., Lubin, L. M., Lemaux, B. C., et al. 2009, ApJ, 700, 901

Kormendy, J., \& Richstone, D. 1995, ARA\&A, 33, 581

Kriss, G. A. 1994, in ASP Conf. Ser. 61, Astronomical Data Analysis Software and Systems III, ed. D. R. Crabtree, R. J. Hanisch, \& J. Barnes (San Francisco, CA: ASP), 437

Krolik, J. H. 1999, Active Galactic Nuclei: From the Central Black Hole to the Galactic Environment (Princeton, NJ: Princeton Univ. Press)

Magorrian, J., Tremaine, S., Richstone, D., et al. 1998, AJ, 115, 2285

Massey, P., Strobel, K., Barnes, J. V., \& Anderson, E. 1988, ApJ, 328, 315

Merritt, D., \& Ferrarese, L. 2001, MNRAS, 320, L30

Oke, J. B., Cohen, J. G., Carr, M., et al. 1995, PASP, 107, 375

Richards, G. T., Hall, P. B., Vanden Berk, D. E., et al. 2003, AJ, 126, 1131

Richstone, D., Ajhar, E. A., Bender, R., et al. 1998, Natur, 395, A14

Runnoe, J. C., Brotherton, M. S., \& Shang, Z. 2012, MNRAS, 422, 478

Sanders, D. B., \& Mirabel, I. F. 1996, ARA\&A, 34, 749

Schawinski, K., Dowlin, N., Thomas, D., Urry, C. M., \& Edmondson, E. 2010a, ApJL, 714, 108

Schawinski, K., Urry, C. M., Virani, S., et al. 2010b, ApJ, 711, 284

Schawinski, K., Virani, S., Simmons, B., et al. 2009, ApJL, 692, 19

Schlegel, D. J., Finkbeiner, D. P., \& Davis, M. 1998, ApJ, 500, 525

Serra, P., \& Trager, S. C. 2007, MNRAS, 374, 769

Shen, Y., Richards, G. T., Strauss, M. A., et al. 2011, ApJS, 194, 45

Springel, V., Di Matteo, T., \& Hernquist, L. 2005, ApJL, 620, 79

Trager, S. C., \& Somerville, R. S. 2009, MNRAS, 395, 608

Tremaine, S., Gebhardt, K., Bender, R., et al. 2002, ApJ, 574, 740

Vanden Berk, D. E., Richards, G. T., Bauer, A., et al. 2001, AJ, 122, 549

Vestergaard, M., \& Osmer, P. S. 2009, ApJ, 699, 800

Vestergaard, M., \& Peterson, B. M. 2006, ApJ, 641, 689

Vestergaard, M., \& Wilkes, B. J. 2001, ApJS, 134, 1

Wild, V., Heckman, T., \& Charlot, S. 2010, MNRAS, 405, 933

Wild, V., Walcher, C. J., Johansson, P. H., et al. 2009, MNRAS, 395, 144

Yan, R., Newman, J. A., Faber, S. M., et al. 2006, ApJ, 648, 281 